\title{
Boundary value problems for the elliptic sine-Gordon equation in a semi-strip
}

Article

Accepted Version

Fokas, A. S., Lenells, J. and Pelloni, B. (2013) Boundary value problems for the elliptic sine-Gordon equation in a semi-strip. Journal of Nonlinear Science, 23 (2). pp. 241-282. ISSN 20081901 doi: https://doi.org/10.1007/s00332-012-9150-5 Available at https://centaur.reading.ac.uk/29139/

It is advisable to refer to the publisher's version if you intend to cite from the work. See Guidance on citing.

To link to this article DOI: http://dx.doi.org/10.1007/s00332-012-9150-5

Publisher: JNSA

All outputs in CentAUR are protected by Intellectual Property Rights law, including copyright law. Copyright and IPR is retained by the creators or other copyright holders. Terms and conditions for use of this material are defined in the End User Agreement.

\section{www.reading.ac.uk/centaur}

\section{CentAUR}

Central Archive at the University of Reading

Reading's research outputs online 


\title{
Boundary value problems for the elliptic sine-Gordon equation in a semi-strip
}

\author{
A.S. Fokas* J. Lenells** and B. Pelloni*** \\ *Department of Applied Mathematics and Theoretical Physics \\ Cambridge University \\ Cambridge CB3 0WA, UK. \\ t.fokas@damtp.cam.ac.uk \\ **Department of Mathematics \\ Baylor University \\ Waco, TX 76798, USA \\ Jonatan_Lenells@baylor.edu \\ ***Department of Mathematics \\ University of Reading \\ Reading RG6 6AX, UK \\ b.pelloni@reading.ac.uk
}

September 4, 2012

\begin{abstract}
We study boundary value problems posed in a semistrip for the elliptic sine-Gordon equation, which is the paradigm of an elliptic integrable PDE in two variables. We use the method introduced by one of the authors, which provides a substantial generalization of the inverse scattering transform and can be used for the analysis of boundary as opposed to initial-value problems. We first express the solution in terms of a $2 \times 2$ matrix Riemann-Hilbert problem whose "jump matrix" depends on both the Dirichlet and the Neumann boundary values. For a well posed problem one of these boundary values is an unknown function. This unknown function is characterised in terms of the so-called global relation, but in general this characterisation is nonlinear. We then concentrate on the case that the prescribed boundary conditions are zero along the unbounded sides of a semistrip and constant along the bounded side. This corresponds to a case of the so-called linearisable boundary conditions, however a major difficulty for this problem is the existence of non-integrable singularities of the function $q_{y}$ at the two corners of the semistrip; these singularities are generated by the discontinuities of the boundary condition at these corners. Motivated by the recent solution of the analogous problem for the modified Helmholtz equation, we introduce an appropriate regularisation which overcomes this difficulty. Furthermore, by mapping the basic Riemann-Hilbert problem to an equivalent modified Riemann-Hilbert problem, we show that the solution can be expressed in terms of a $2 \times 2$ matrix Riemann-Hilbert problem whose "jump matrix" depends explicitly on the width of the semistrip $L$, on the constant value $d$ of the solution along the bounded side, and on the residues at the given poles of a certain spectral function denoted by $h(\lambda)$. The determination of the function $h$ remains open.
\end{abstract}




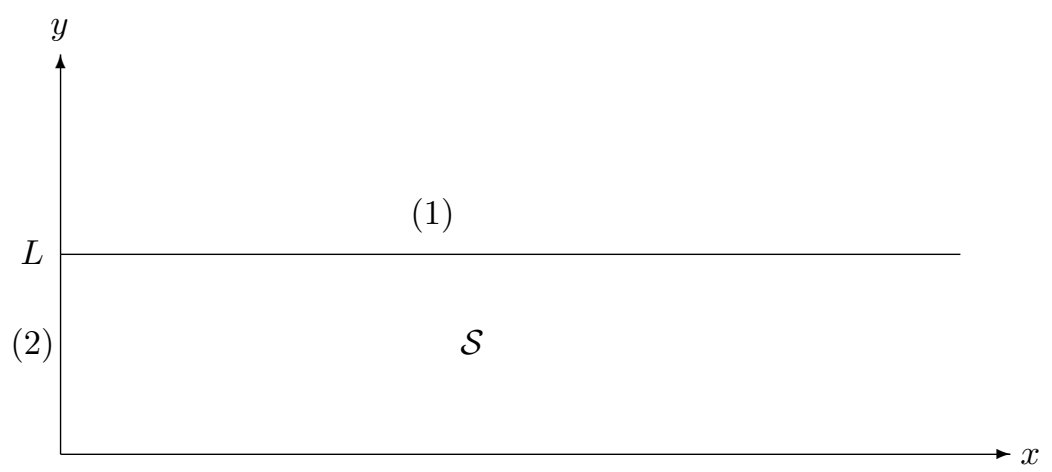

(3)

Figure 1: The semistrip S

\section{Introduction}

A method for solving initial-boundary value problems for linear and integrable nonlinear PDEs was introduced in $[13,14]$ and developed by several authors, see the survey [15] and the references therein. This method has already been used for:

(a) linear and integrable nonlinear evolution PDEs formulated on the half line and on a finite interval $[4,5,11,12,17,20,21,22,23,24,27,38,39,47]$;

(b) linear and integrable nonlinear hyperbolic PDEs [26, 40, 42];

(c) linear elliptic PDEs $[1,3,8,9,16,28,29,44,45,46]$.

The aim of this paper is to implement this method in the case of the prototypical integrable nonlinear elliptic PDE, namely the celebrated elliptic sine-Gordon equation. This equation was first analysed in [37] (see also [7, 30]); simple boundary value problems for this equation, using the method of [13], have been considered in [41, 43]). For the case of nonlinear elliptic PDEs in cylindrical coordinates, see [32, 34].

We will consider the sine-Gordon equation in the form

$$
q_{x x}+q_{y y}=\sin q, \quad q=q(x, y),
$$

and we will analyze boundary value problems posed in the semi-infinite strip

$$
\mathcal{S}=\{0<x<\infty, \quad 0<y<L\},
$$

where $L$ is a positive finite constant. The sides $\{y=L, 0<x<\infty\},\{x=0,0<y<L\}$ and $\{y=0,0<x<\infty\}$ will be referred to as side (1), (2) and (3) respectively, see figure 1.

Suppose that (1.1) is supplemented with appropriate, compatible boundary conditions on the boundary of the semistrip $\mathcal{S}$, so that there exists a unique solution $q(x, y)$. It will be shown in section 2 that this solution can be expressed in terms of the solution of a $2 \times 2$ matrix RiemannHilbert $(\mathrm{RH})$ problem with a jump on the union of the real and imaginary axis of the $\lambda$ complex plane. The "jump matrix" is expressed in terms of certain functions, called spectral functions, which will be denoted by $\left\{a_{j}(\lambda), b_{j}(\lambda)\right\}, j=1,2,3$. These functions can be uniquely characterized via the solution of certain linear Volterra integral equations, in terms of the Dirichlet and Neumann boundary values. Namely, $\left\{a_{1}, b_{1}\right\},\left\{a_{2}, b_{2}\right\}$ and $\left\{a_{3}, b_{3}\right\}$ are uniquely determined in terms of $\left\{q(x, L), q_{y}(x, L)\right\},\left\{q(0, y), q_{x}(0, y)\right\}$ and $\left\{q(x, 0), q_{y}(x, 0)\right\}$ respectively. However, for a well posed problem only a subset of these boundary values are prescribed as boundary conditions. Thus, in order to compute the spectral functions in terms of the given boundary conditions, one must first 
determine the unknown boundary values, i.e. one must characterize the Dirichlet to Neumann map. The solution of this problem, which makes crucial use of the so-called global relation, yields in general a nonlinear map, see $[6,18,25,35,36]$.

In the case of integrable nonlinear evolution PDEs, it has been shown in $[17,19,23,24,33]$ that there exists a particular class of boundary conditions, called linearizable, for which it is possible to avoid the above nonlinear map. The main result of the present paper is the analysis of a particular case of linearizable boundary conditions for the sine-Gordon equation on the semi-infinite strip. In particular, the following boundary conditions will be investigated in detail:

$$
q(x, L)=q(x, 0)=0, \quad 0<x<\infty ; \quad q(0, y)=d, \quad 0<y<L,
$$

where $d$ is a finite constant. We assume that $0<d<\pi$. These boundary conditions are discontinuous at the corners $(0,0)$ and $(0, L)$ of the domain. This implies that $q_{y}(x, y)$ has a non-integrable singularity at the two corners of the semistrip. Using an appropriate gauge transformation, which is motivated by the recent solution of the analogous problem for the modified Helmholtz equation ([2], see also Appendix A), we are able to overcome this difficulty and introduce well-defined spectral functions. Furthermore, we show that the basic Riemann-Hilbert problem can be mapped to a simpler Riemann-Hilbert problem whose jump matrix, instead of depending on the six unknown spectral functions $\left\{a_{j}(\lambda), b_{j}(\lambda)\right\}_{1}^{3}$, depends explicitly on the given constant $d$, on the width $L$ of the semistrip, and on the residues at the given poles of a certain spectral function, denoted by $h(\lambda)$. The rigorous analysis for the determination of $h$ remains open.

This result, as well as the analogous result valid for the elliptic version of the Ernst equation $[32,34]$, imply that the method of [13] provides a powerful tool for analyzing effectively a large class of interesting boundary conditions.

\section{Spectral analysis under the assumption of existence}

In what follows we assume that (1.1) is supplemented with appropriate boundary conditions on the boundary of the semistrip $\mathcal{S}$, compatible at the corners of the domain, so that the existence of a unique, smooth solution $q(x, y)$ can be assumed. Furthermore, we assume the following:

$$
\begin{aligned}
& q(x, L), q_{y}(x, L), q(x, 0), q_{y}(x, 0) \in \mathbf{L}^{1}\left(\mathbb{R}^{+}\right), \\
& x q(x, L), x q_{y}(x, L), x q(x, 0), x q_{y}(x, 0) \in \mathbf{L}^{1}\left(\mathbb{R}^{+}\right), \\
& q(0, y), q_{x}(0, y), y q(0, y), y q_{x}(0, y) \in \mathbf{L}^{1}([0, L]) .
\end{aligned}
$$

The sine-Gordon equation is the compatibility condition of the following Lax pair [31] for the $2 \times 2$ matrix-valued function $\Psi(x, y, \lambda), \lambda \in \mathbb{C}$ :

$$
\begin{aligned}
& \Psi_{x}+\frac{\Omega(\lambda)}{2}\left[\sigma_{3}, \Psi\right]=Q(x, y, \lambda) \Psi, \\
& \Psi_{y}+\frac{\omega(\lambda)}{2}\left[\sigma_{3}, \Psi\right]=i Q(x, y,-\lambda) \Psi,
\end{aligned}
$$

where

$$
\Omega(\lambda)=\frac{1}{2 i}\left(\lambda-\frac{1}{\lambda}\right), \quad \omega(\lambda)=\frac{1}{2}\left(\lambda+\frac{1}{\lambda}\right)
$$



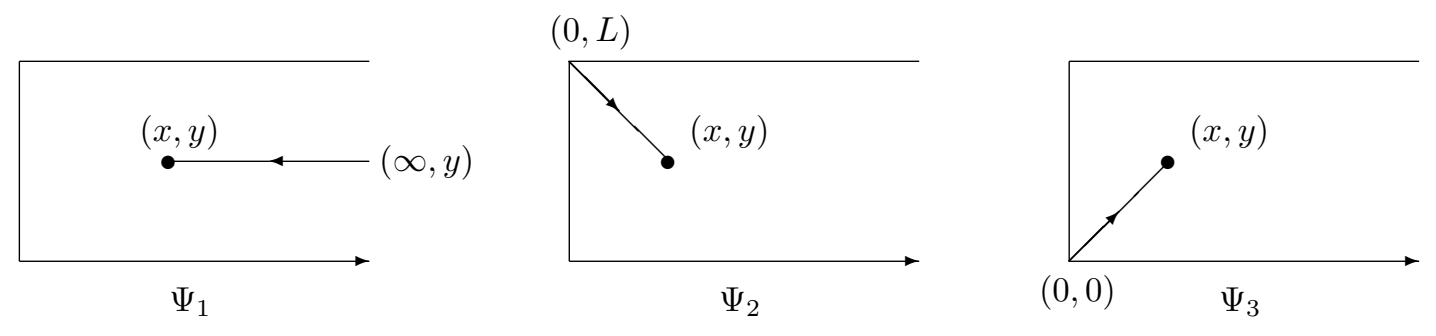

Figure 2: The functions $\Psi_{1}, \Psi_{2}$ and $\Psi_{3}$

$$
Q(x, y, \lambda)=\frac{i}{4}\left(\begin{array}{cc}
\frac{1}{\lambda}(1-\cos q) & q_{x}-i q_{y}+\frac{i \sin q}{\lambda} \\
q_{x}-i q_{y}-\frac{i \sin q}{\lambda} & -\frac{1}{\lambda}(1-\cos q)
\end{array}\right), \quad q=q(x, y) .
$$

Equations (2.2) and (2.3) can be written as the single equation

$$
d\left(\mathrm{e}^{(\Omega(\lambda) x+\omega(\lambda) y) \frac{\widehat{\frac{\sigma}{3}}}{2}} \Psi(x, y, \lambda)\right)=W(x, y, \lambda),
$$

where the differential form $W$ is given by

$$
W(x, y, \lambda)=\mathrm{e}^{(\Omega(\lambda) x+\omega(\lambda) y) \frac{\widehat{\sigma_{3}}}{2}}(Q(x, y, \lambda) \Psi(x, y, \lambda) d x+i Q(x, y,-\lambda) \Psi(x, y, \lambda) d y),
$$

and $\widehat{\sigma_{3}}$ acts on a $2 \times 2$ matrix $A$ by

$$
\widehat{\sigma_{3}} A=\left[\sigma_{3}, A\right] .
$$

Remark 2.1 Note that

$$
\overline{\Omega(\bar{\lambda})}=-\Omega(\lambda)=\Omega\left(\frac{1}{\lambda}\right), \quad \overline{\omega(\bar{\lambda})}=\omega(\lambda)=\omega\left(\frac{1}{\lambda}\right) .
$$

\subsection{Bounded and analytic eigenfunctions}

We define three solutions $\Psi_{j}(x, y, \lambda), j=1,2,3$, of (2.6) by

$$
\Psi_{j}(x, y, \lambda)=I+\int_{\left(x_{j}, y_{j}\right)}^{(x, y)} \mathrm{e}^{-(\Omega(\lambda) x+\omega(\lambda) y) \frac{\widehat{\sigma}}{2}} W(\xi, \eta, \lambda),
$$

where

$$
\left(x_{1}, y_{1}\right)=(\infty, y), \quad\left(x_{2}, y_{2}\right)=(0, L), \quad\left(x_{3}, y_{3}\right)=(0,0) .
$$

Since the differential form $W$ is exact, the integral on the right hand side of (2.8) is independent of the path of integration. We choose the particular contours shown in figure 2. This choice implies the following inequalities on the contours:

$$
\left(x_{1}, y_{1}\right) \rightarrow(x, y): \quad \xi-x \geq 0,
$$




$$
\begin{aligned}
& \left(x_{2}, y_{2}\right) \rightarrow(x, y): \quad \xi-x \leq 0, \eta-y \geq 0 \\
& \left(x_{3}, y_{3}\right) \rightarrow(x, y): \quad \xi-x \leq 0, \eta-y \leq 0 .
\end{aligned}
$$

The first inequality above implies that the exponential appearing in the second (first) column of the right hand side of the equation defining $\Psi_{1}$ is bounded and analytic for $\operatorname{Im}(\lambda)<0(\operatorname{Im}(\lambda)>0)$. Similar considerations are valid for $\Psi_{2}$ and $\Psi_{3}$. Hence we denote the matrices $\Psi_{j}$ as follows:

$$
\Psi_{1}=\left(\Psi_{1}^{(12)}, \Psi_{1}^{(34)}\right), \quad \Psi_{2}=\left(\Psi_{2}^{(4)}, \Psi_{2}^{(2)}\right), \quad \Psi_{3}=\left(\Psi_{3}^{(3)}, \Psi_{3}^{(1)}\right),
$$

where the superscript (12) denotes the union of the first and second quadrants of the $\lambda$ complex

plane, and similarly for the other superscripts. The function $\Psi_{1}^{(12)}$ is analytic for $\operatorname{Im}(\lambda)>0$ and it has essential singularities at $\lambda=\infty$ and $\lambda=0$; furthermore,

$$
\Psi_{1}^{(12)}=\left(\begin{array}{l}
1 \\
0
\end{array}\right)+O\left(\frac{1}{\lambda}\right), \quad \lambda \rightarrow \infty, \quad \operatorname{Im}(\lambda) \geq 0 .
$$

Similar considerations are valid for the column vectors $\Psi_{1}^{(34)}, \Psi_{3}^{(3)}$ and $\Psi_{3}^{(1)}$. The function $\Psi_{2}$ is an analytic function in the entire complex plane, except at $\lambda=\infty$ and $\lambda=0$, where it has essential singularities. In addition,

$$
\begin{array}{ll}
\Psi_{2}^{(4)}=\left(\begin{array}{l}
1 \\
0
\end{array}\right)+O\left(\frac{1}{\lambda}\right), \quad \lambda \rightarrow \infty, \quad \frac{3 \pi}{2} \leq \arg (\lambda) \leq 2 \pi, \\
\Psi_{2}^{(2)}=\left(\begin{array}{l}
0 \\
1
\end{array}\right)+O\left(\frac{1}{\lambda}\right), \quad \lambda \rightarrow \infty, \quad \frac{\pi}{2} \leq \arg (\lambda) \leq \pi .
\end{array}
$$

\subsection{Spectral functions}

Any two solutions $\Psi, \tilde{\Psi}$ of (2.6) are related by an equation of the form

$$
\Psi(x, y, \lambda)=\tilde{\Psi}(x, y, \lambda) \mathrm{e}^{-(\Omega(\lambda) x+\omega(\lambda) y) \frac{\widehat{\sigma_{3}}}{2}} C(\lambda) .
$$

We introduce the notations

$$
S_{1}(\lambda)=\Psi_{1}(0, L, \lambda), \quad S_{2}(\lambda)=\Psi_{2}(0,0, \lambda), \quad S_{3}(\lambda)=\Psi_{1}(0,0, \lambda) .
$$

Then equation (2.12) implies the following equations:

$$
\begin{array}{ll}
\Psi_{1}(x, y, \lambda)=\Psi_{2}(x, y, \lambda) \mathrm{e}^{-(\Omega(\lambda) x+\omega(\lambda) y) \frac{\widehat{\sigma_{3}}}{2}} \mathrm{e}^{\frac{\omega(\lambda)}{2} L \widehat{\sigma_{3}}} S_{1}(\lambda), \quad \lambda \in \mathbb{R}, \\
\Psi_{2}(x, y, \lambda)=\Psi_{3}(x, y, \lambda) \mathrm{e}^{-(\Omega(\lambda) x+\omega(\lambda) y) \frac{\widehat{\sigma_{3}}}{2}} S_{2}(\lambda), \quad \lambda \in \mathbb{C} \backslash\{0\}, \\
\Psi_{1}(x, y, \lambda)=\Psi_{3}(x, y, \lambda) \mathrm{e}^{-(\Omega(\lambda) x+\omega(\lambda) y) \frac{\widehat{\sigma_{3}}}{2}} S_{3}(\lambda), \quad \lambda \in\left(\mathbb{R}^{-}, \mathbb{R}^{+}\right) .
\end{array}
$$

The notation $\lambda \in\left(\mathbb{R}^{-}, \mathbb{R}^{+}\right)$means that the equation for the first column vector in (2.16) is valid for $\lambda \in \mathbb{R}^{-}$, while the equation for the second vector is valid for $\mathbb{R}^{+}$.

Equations (2.13)-(2.16) suggest the following definitions:

$$
S_{1}(\lambda)=\Phi_{1}(0, \lambda), \quad \Phi_{1}(x, \lambda)=I-\int_{x}^{\infty} \mathrm{e}^{\Omega(\lambda)(\xi-x) \frac{\widehat{\sigma}}{2}} Q(\xi, L, \lambda) \Phi_{1}(\xi, \lambda) d \xi
$$




$$
\begin{array}{ll}
\lambda \in\left(\mathbb{C}^{+}, \mathbb{C}^{-}\right), \quad 0<x<\infty, \\
S_{2}(\lambda)=\Phi_{2}(0, \lambda), \quad \Phi_{2}(y, \lambda)=I-i \int_{y}^{L} \mathrm{e}^{\omega(\lambda)(\eta-y) \frac{\widehat{\sigma_{3}}}{2}} Q(0, \eta,-\lambda) \Phi_{2}(\eta, \lambda) d \eta, \\
\lambda \in \mathbb{C}, \quad 0<y<L, \\
S_{3}(\lambda)=\Phi_{3}(0, \lambda), \quad \Phi_{3}(x, \lambda)=I-\int_{x}^{\infty} \mathrm{e}^{\Omega(\lambda)(\xi-x) \frac{\widehat{\sigma_{3}}}{2}} Q(\xi, 0, \lambda) \Phi_{3}(\xi, \lambda) d \xi, \\
\lambda \in\left(\mathbb{C}^{+}, \mathbb{C}^{-}\right), \quad 0<x<\infty .
\end{array}
$$

The matrix $Q$ satisfies the symmetry properties

$$
Q(\lambda)_{22}=Q(-\lambda)_{11}, \quad Q(\lambda)_{12}=Q(-\lambda)_{21}
$$

Hence the matrices $\Phi_{i}, i=1, . ., 3$, can be represented in the form

$$
\Phi_{1}=\left(\begin{array}{cc}
A_{1}(x, \lambda) & B_{1}(x,-\lambda) \\
B_{1}(x, \lambda) & A_{1}(x,-\lambda)
\end{array}\right), \quad \Phi_{2}=\left(\begin{array}{cc}
A_{2}(y, \lambda) & B_{2}(y,-\lambda) \\
B_{2}(y, \lambda) & A_{2}(y,-\lambda)
\end{array}\right), \quad \Phi_{3}=\left(\begin{array}{cc}
A_{3}(x, \lambda) & B_{3}(x,-\lambda) \\
B_{3}(x, \lambda) & A_{3}(x,-\lambda)
\end{array}\right),
$$

and therefore

$$
S_{i}(\lambda)=\left(\begin{array}{cc}
a_{i}(\lambda) & b_{i}(-\lambda) \\
b_{i}(\lambda) & a_{i}(-\lambda)
\end{array}\right), \quad i=1,2,3 .
$$

The spectral functions $\left\{a_{1}(\lambda), b_{1}(\lambda)\right\},\left\{a_{2}(\lambda), b_{2}(\lambda)\right\}$ and $\left\{a_{3}(\lambda), b_{3}(\lambda)\right\}$ are defined in terms of $\left\{q(x, L), q_{y}(x, L)\right\},\left\{q(0, y), q_{x}(0, y)\right\}$ and $\left\{q(x, 0), q_{y}(x, 0)\right\}$ respectively, through equations (2.17)-(2.19).

These functions have the following properties:

- $a_{1}(\lambda), b_{1}(\lambda)$ are analytic and bounded in $\mathbb{C}^{+}$.

$a_{1}(\lambda) a_{1}(-\lambda)-b_{1}(\lambda) b_{1}(-\lambda)=1, \lambda \in \mathbb{R}$.

$a_{1}(\lambda)=1+O\left(\frac{1}{\lambda}\right), b_{1}(\lambda)=O\left(\frac{1}{\lambda}\right)$ as $\lambda \rightarrow \infty, \operatorname{Im}(\lambda) \geq 0$.

- $a_{2}(\lambda), b_{2}(\lambda)$ are analytic functions of $\lambda$ for all $\lambda \in \mathbb{C}$, except for essential singularities at $\lambda=\infty$ and $\lambda=0$.

$a_{2}(\lambda) a_{2}(-\lambda)-b_{2}(\lambda) b_{2}(-\lambda)=1, \lambda \in \mathbb{C} \backslash\{0\}$.

$a_{2}(\lambda)=1+O\left(\frac{1}{\lambda}\right), b_{2}(\lambda)=O\left(\frac{1}{\lambda}\right)$ as $\lambda \rightarrow \infty, \frac{3 \pi}{2} \leq \arg (\lambda) \leq 2 \pi$.

- $a_{3}(\lambda), b_{3}(\lambda)$ are analytic and bounded in $\mathbb{C}^{+}$.

$a_{3}(\lambda) a_{3}(-\lambda)-b_{3}(\lambda) b_{3}(-\lambda)=1, \lambda \in \mathbb{R}$.

$a_{3}(\lambda)=1+O\left(\frac{1}{\lambda}\right), b_{3}(\lambda)=O\left(\frac{1}{\lambda}\right)$ as $\lambda \rightarrow \infty, \operatorname{Im}(\lambda) \geq 0$.

These properties follow from the analogous properties of the matrix-valued functions $\Phi_{j}, j=1,2,3$, from the condition of unit determinant, and from the large $\lambda$ asymptotics of these functions. 


\subsection{The global relation}

Evaluating equations (2.15) and (2.16) at $x=0, y=L$, we find

$$
I=\Psi_{3}(0, L, \lambda) \mathrm{e}^{-\frac{\omega(\lambda)}{2} L \widehat{\sigma_{3}}} S_{2}(\lambda)
$$

and

$$
S_{1}(\lambda)=\Psi_{3}(0, L, \lambda) \mathrm{e}^{-\frac{\omega(\lambda)}{2} L \widehat{\sigma_{3}}} S_{3}(\lambda) .
$$

Eliminating $\Psi_{3}(0, L, \lambda)$ we obtain

$$
\mathrm{e}^{\frac{\omega(\lambda)}{2} L \widehat{\sigma_{3}}} S_{1}(\lambda)=S_{2}(\lambda)^{-1} S_{3}(\lambda) .
$$

The first column vector of this equation yields the following global relations:

$$
\begin{array}{ccc}
a_{1}(\lambda)=a_{2}(-\lambda) a_{3}(\lambda)-b_{2}(-\lambda) b_{3}(\lambda), & & \lambda \in \mathbb{C}^{+}, \\
b_{1}(\lambda) \mathrm{e}^{-\omega(\lambda) L}=a_{2}(\lambda) b_{3}(\lambda)-a_{3}(\lambda) b_{2}(\lambda), & & \lambda \in \mathbb{C}^{+} .
\end{array}
$$

\subsection{The Riemann-Hilbert problem}

Equations (2.14)-(2.16), relating the various analytic eigenfunctions, can be rewritten in a form that determines the jump conditions of a $2 \times 2 \mathrm{RH}$ problem, with unitary jump matrices on the real and imaginary axes. This involves tedious but straightforward algebraic manipulations. The final form is

$$
M_{-}(x, y, \lambda)=M_{+}(x, y, \lambda) J(x, y, \lambda), \quad \lambda \in \mathbb{R} \cup i \mathbb{R},
$$

where the matrices $M_{ \pm}$and $J$ are defined as follows:

$$
\begin{gathered}
M_{+}=\left(\Psi_{1}^{(12)}, \frac{1}{a_{3}(\lambda)} \Psi_{3}^{(1)}\right), \quad \arg (\lambda) \in\left[0, \frac{\pi}{2}\right], \\
M_{-}=\left(\Psi_{1}^{(12)}, \frac{1}{a_{1}(\lambda)} \Psi_{2}^{(2)}\right), \quad \arg (\lambda) \in\left[\frac{\pi}{2}, \pi\right], \\
M_{+}=\left(\frac{1}{a_{3}(-\lambda)} \Psi_{3}^{(3)}, \Psi_{1}^{(34)}\right), \quad \arg (\lambda) \in\left[\pi, \frac{3 \pi}{2}\right], \\
M_{-}=\left(\frac{1}{a_{1}(-\lambda)} \Psi_{2}^{(4)}, \Psi_{1}^{(34)}\right), \quad \arg (\lambda) \in\left[\frac{3 \pi}{2}, 2 \pi\right], \\
J(x, y, \lambda)=J^{\alpha}(x, y, \lambda), \text { if } \arg (\lambda)=\alpha, \quad \alpha=0, \frac{\pi}{2}, \pi, \frac{3 \pi}{2},
\end{gathered}
$$

where, using the global relations (2.22), we find

$$
\begin{gathered}
J^{0}=\left(\begin{array}{cc}
\frac{a_{2}(\lambda)}{a_{1}(-\lambda) a_{3}(\lambda)} & \frac{b_{3}(-\lambda)}{a_{3}(\lambda)} \mathrm{e}^{-\theta(x, y, \lambda)} \\
-\frac{\mathrm{e}^{-\omega(\lambda) L} b_{1}(\lambda)}{a_{1}(-\lambda)} \mathrm{e}^{\theta(x, y, \lambda)} & 1
\end{array}\right), \\
J^{\pi / 2}=\left(\begin{array}{cc}
1 & \frac{b_{2}(-\lambda)}{a_{1}(\lambda) a_{3}(\lambda)} \mathrm{e}^{-\theta(x, y, \lambda)} \\
0 & 1
\end{array}\right), \quad J^{3 \pi / 2}=\left(\begin{array}{cc}
1 & 0 \\
\frac{b_{2}(\lambda)}{a_{1}(-\lambda) a_{3}(-\lambda)} \mathrm{e}^{\theta(x, y, \lambda)} & 1
\end{array}\right)
\end{gathered}
$$


and

$$
J^{\pi}=J^{3 \pi / 2}\left(J^{0}\right)^{-1} J^{\pi / 2},
$$

where

$$
\theta(x, y, \lambda)=\Omega(\lambda) x+\omega(\lambda) y .
$$

All the matrices $J^{\alpha}$ have unit determinant: for $J^{\pi / 2}$ and $J^{3 \pi / 2}$ this is immediate, whereas for $J^{0}$ we find

$$
\operatorname{det}\left(J^{0}\right)=\frac{a_{2}(\lambda)+\mathrm{e}^{-\omega(\lambda) L} b_{1}(\lambda) b_{3}(-\lambda)}{a_{1}(-\lambda) a_{3}(\lambda)}=\frac{a_{1}(-\lambda) a_{3}(\lambda)}{a_{1}(-\lambda) a_{3}(\lambda)}=1,
$$

where we have used the equation

$$
a_{2}(\lambda)=a_{1}(-\lambda) a_{3}(\lambda)-b_{3}(-\lambda) b_{1}(\lambda) \mathrm{e}^{-\omega(\lambda) L}, \quad \lambda \in \mathbb{R},
$$

which is a consequence of the global relations (2.22).

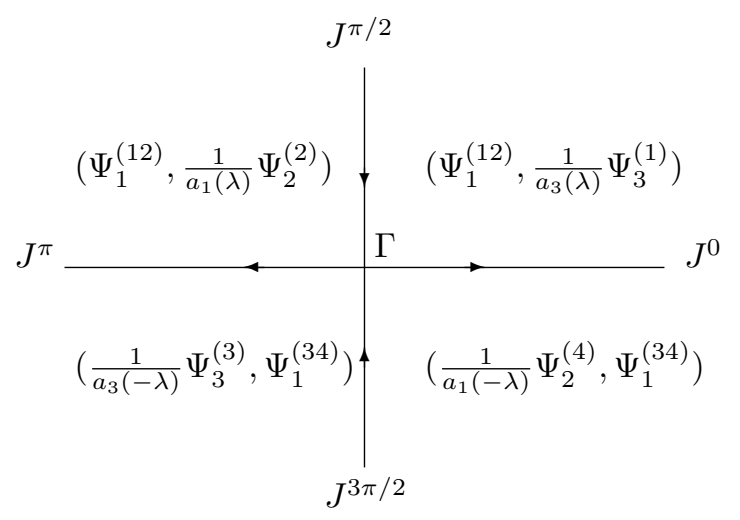

Figure 3: The bounded eigenfunctions and the Riemann-Hilbert problem

The solution $M(x, y, \lambda)$ of this $\mathrm{RH}$ problem is a sectionally meromorphic function of $\lambda$. The possible poles of this function are generated by the zeros of the function $a_{1}(\lambda)$ in the region $\left\{\arg (\lambda) \in\left[\frac{\pi}{2}, \pi\right]\right\}$, by the zeros of $a_{3}(\lambda)$ in the region $\left\{\arg (\lambda) \in\left[0, \frac{\pi}{2}\right]\right\}$, and by the corresponding zeros of $a_{1}(-\lambda), a_{3}(-\lambda)$.

We assume

- The possible zeros of $a_{1}$ in the region $\left\{\arg (\lambda) \in\left(\frac{\pi}{2}, \pi\right)\right\}$ are simple; these zeros are denoted $\lambda_{j}, j=1, . ., N_{1}$

- The possible zeros of $a_{3}$ in the region $\left\{\arg (\lambda) \in\left(0, \frac{\pi}{2}\right)\right\}$ are simple; these zeros are denoted $\zeta_{j}, j=1, . ., N_{3}$

The residues of the function $M$ at the corresponding poles can be computed using equations (2.14)-(2.16). Indeed, equation (2.16) yields

$$
\Psi_{1}^{(12)}=a_{3} \Psi_{3}^{(3)}+b_{3} \mathrm{e}^{\theta(x, y, \lambda)} \Psi_{3}^{(1)},
$$


hence

$$
\operatorname{Res}_{\zeta_{j}} \frac{\Psi_{3}^{(1)}}{a_{3}}=\frac{\Psi_{3}^{(1)}\left(\zeta_{j}\right)}{\dot{a}_{3}\left(\zeta_{j}\right)}=\frac{\Psi_{1}^{(12)}\left(\zeta_{j}\right)}{\dot{a}_{3}\left(\zeta_{j}\right) b_{3}\left(\zeta_{j}\right)} \mathrm{e}^{-\theta\left(x, y, \zeta_{j}\right)},
$$

where $\dot{a}_{3}(\lambda)$ denotes the derivative of $a_{3}$ with respect to $\lambda$.

Similarly, using (2.14),

$$
\operatorname{Res}_{\lambda_{j}} \frac{\Psi_{2}^{(2)}}{a_{1}}=\frac{\Psi_{2}^{(2)}\left(\lambda_{j}\right)}{\dot{a}_{1}\left(\lambda_{j}\right)}=\frac{\Psi_{1}^{(12)}\left(\lambda_{j}\right)}{\dot{a}_{1}\left(\lambda_{j}\right) b_{1}\left(\lambda_{j}\right) \mathrm{e}^{-\omega\left(\lambda_{j}\right) L}} \mathrm{e}^{-\theta\left(x, y, \lambda_{j}\right)} .
$$

Using the notation $[M]_{1}$ for the first column and $[M]_{2}$ for the second column of the solution $M$ of the $\mathrm{RH}$ problem (2.23), the equations (2.30) and (2.31) imply the following residue conditions:

$$
\begin{aligned}
\operatorname{Res}_{\zeta_{j}}[M(x, y, \lambda)]_{2} & =\frac{\mathrm{e}^{-\theta\left(x, y, \zeta_{j}\right)}}{\dot{a}_{3}\left(\zeta_{j}\right) b_{3}\left(\zeta_{j}\right)}\left[M\left(x, y, \zeta_{j}\right)\right]_{1}, \quad 0<\arg \lambda<\frac{\pi}{2}, \\
\operatorname{Res}_{\lambda_{j}}[M(x, y, \lambda)]_{2} & =\frac{\mathrm{e}^{-\theta\left(x, y, \lambda_{j}\right)}}{\dot{a}_{1}\left(\lambda_{j}\right) b_{1}\left(\lambda_{j}\right) \mathrm{e}^{-\omega\left(\lambda_{j}\right) L}}\left[M\left(x, y, \lambda_{j}\right)\right]_{1}, \quad \frac{\pi}{2}<\arg \lambda<\pi .
\end{aligned}
$$

Similar residue conditions are obtained in $\mathbb{C}^{-}$by letting $\lambda \rightarrow-\lambda$.

\section{The inverse problem}

Rewriting the jump condition, we obtain

$$
M_{+}-M_{-}=M_{+}-M_{+} J=M_{+}(I-J) \Rightarrow M_{+}-M_{-}=M_{+} \tilde{J},
$$

where $\tilde{J}=I-J$. The asymptotic conditions (2.10)-(2.11) imply

$$
M(x, y, \lambda)=I+\frac{M^{*}(x, y)}{\lambda}+O\left(\frac{1}{\lambda^{2}}\right), \quad|\lambda| \rightarrow \infty, \quad \lambda \in \mathbb{C} \backslash(\mathbb{R} \cup i \mathbb{R}) .
$$

Equation (2.33) and the condition (2.34) yield the following integral representation for the function $M$ :

$$
M(x, y, \lambda)=I+\frac{1}{2 \pi i} \int_{\Gamma} \frac{M_{+}\left(x, y, \lambda^{\prime}\right) \tilde{J}\left(x, y, \lambda^{\prime}\right)}{\lambda^{\prime}-\lambda} d \lambda^{\prime}, \quad \lambda \in \mathbb{C} \backslash \Gamma
$$

where

$$
\Gamma=\mathbb{R} \cup i \mathbb{R}
$$

Equations (2.34) and (2.35) imply

$$
M^{*}=-\frac{1}{2 \pi i} \int_{\Gamma} M_{+}(x, y, \lambda) \tilde{J}(x, y, \lambda) d \lambda .
$$

Using (2.34) in the first ODE in the Lax pair (2.2), we find

$$
-\frac{i}{4}\left[\sigma_{3}, M^{*}\right]=i \frac{q_{x}-i q_{y}}{4} \sigma_{1} \Rightarrow q_{x}-i q_{y}=2\left(M^{*}\right)_{21}=2 \lim _{\lambda \rightarrow \infty}\left(\lambda M_{21}\right),
$$

where $\sigma_{1}, \sigma_{3}$ denote the usual Pauli matrices.

In order to obtain an expression in terms of $q$ rather than its derivatives, we consider the coefficient in $(2.2)$ of the term $\lambda^{-1}$. The $(1,1)$ element of this coefficient yields

$$
\cos q(x, y)=1+4 i\left(M_{x}^{*}\right)_{11}+2\left(M^{*}\right)_{21}^{2} .
$$




\section{Spectral theory assuming the validity of the global rela- tion}

\subsection{The spectral functions}

The above analysis motivates the following definitions for the spectral functions.

The spectral functions at the $y=0$ and $y=L$ boundaries

Definition 3.1 Given the functions $q(x, L), q_{y}(x, L)$ satisfying conditions (2.1), define the map

$$
\mathbb{S}_{1}:\left\{q(x, L), q_{y}(x, L)\right\} \rightarrow\left\{a_{1}(\lambda), b_{1}(\lambda)\right\}
$$

by

$$
\left(\begin{array}{l}
a_{1}(\lambda) \\
b_{1}(\lambda)
\end{array}\right)=\left[\Phi_{1}(0, \lambda)\right]_{1}, \quad \lambda \in \mathbb{C}^{+},
$$

where $\left[\Phi_{1}(x, \lambda)\right]_{1}$ denotes the first column vector of the unique solution $\Phi_{1}(x, \lambda)$ of the Volterra linear integral equation

$$
\Phi(x, \lambda)=I-\int_{x}^{\infty} \mathrm{e}^{\Omega(\lambda)(\xi-x) \frac{\widehat{\sigma_{3}}}{2}} Q(\xi, L, \lambda) \Phi(\xi, \lambda) d \xi
$$

and $Q(x, L, \lambda)$ is given in terms of $q(x, L)$ and $q_{y}(x, L)$ by equation (2.5).

In what follows, we also assume that the function $a_{1}(\lambda)$ may have $N_{1}$ simple poles $\lambda_{j}$ in $\mathbb{C}^{+}$. Similarly for $a_{3}(\lambda)$.

Proposition 3.1 The spectral functions $a_{1}(\lambda), b_{1}(\lambda)$ have the following properties.

(i) $a_{1}(\lambda), b_{1}(\lambda)$ are continuous and bounded for $\operatorname{Im}(\lambda) \geq 0$, and analytic for $\operatorname{Im}(\lambda)>0$.

(ii) $a_{1}(\lambda)=1+O\left(\frac{1}{\lambda}\right), b_{1}(\lambda)=O\left(\frac{1}{\lambda}\right)$ as $\lambda \rightarrow \infty, \operatorname{Im}(\lambda) \geq 0$.

(iii) $a_{1}(\lambda)=\cos \frac{q(0, L)}{2}+O(\lambda), b_{1}(\lambda)=i \sin \frac{q(0, L)}{2}+O(\lambda)$ as $\lambda \rightarrow 0, \operatorname{Im}(\lambda) \geq 0$.

(iv) $a_{1}(\lambda) a_{1}(-\lambda)-b_{1}(\lambda) b_{1}(-\lambda)=1, \lambda \in \mathbb{R}$.

(v) The map $\mathbb{Q}_{1}:\left\{a_{1}, b_{1}\right\} \rightarrow\left\{q(x, L) q_{y}(x, L)\right\}$, inverse to $\mathbb{S}_{1}$, is given

$$
\begin{aligned}
\cos q(x, L) & =1+4 i \lim _{\lambda \rightarrow \infty}\left(\lambda M_{x}\right)_{11}+2\left(\lim _{\lambda \rightarrow \infty}(\lambda M)_{21}\right)^{2}, \\
q_{y}(x, L) & =-i q_{x}(x, L)+2 i \lim _{\lambda \rightarrow \infty}(\lambda M)_{21},
\end{aligned}
$$

where $M$ is the solution of the following Riemann-Hilbert problem:

- The function

$$
M(x, \lambda)= \begin{cases}M_{+}(x, \lambda) & \lambda \in \mathbb{C}^{+} \\ M_{-}(x, \lambda) & \lambda \in \mathbb{C}^{-}\end{cases}
$$

is a sectionally meromorphic function of $\lambda \in \mathbb{C}$. 
- $M=I+O\left(\frac{1}{\lambda}\right)$ as $\lambda \rightarrow \infty$, and

$$
M_{-}(x, \lambda)=M_{+}(x, \lambda) J_{1}(x, \lambda), \quad \lambda \in \mathbb{R},
$$

where

$$
J_{1}(x, \lambda)=\left(\begin{array}{cc}
1 & -\frac{b_{1}(-\lambda)}{a_{1}(\lambda)} \mathrm{e}^{-\Omega(\lambda) x} \\
\frac{b_{1}(\lambda)}{a_{1}(-\lambda)} \mathrm{e}^{\Omega(\lambda) x} & \frac{1}{a_{1}(\lambda) a_{1}(-\lambda)}
\end{array}\right), \quad \lambda \in \mathbb{R} .
$$

- Let $[M]_{i}$ denote the $i$-th column vector of $M, 1=1,2$. The possible poles of $M_{+}$occur at $\lambda_{j}$, and the possible poles of $M_{-}$occur at $-\lambda_{j}$ in $\mathbb{C}^{-}$, and the associated residues are given by

$$
\begin{aligned}
\operatorname{Res}_{\lambda_{j}}[M(x, \lambda)]_{2} & =\frac{\mathrm{e}^{-\Omega\left(\lambda_{j}\right) x}}{\dot{a}_{1}\left(\lambda_{j}\right) b_{1}\left(\lambda_{j}\right)}\left[M\left(x, \lambda_{j}\right)\right]_{1}, \\
\operatorname{Res}_{-\lambda_{j}}[M(x, \lambda)]_{1} & =\frac{\mathrm{e}^{\Omega\left(\lambda_{j}\right) x}}{\dot{a}_{1}\left(-\lambda_{j}\right) b_{1}\left(-\lambda_{j}\right)}\left[M\left(x,-\lambda_{j}\right)\right]_{2} .
\end{aligned}
$$

The spectral functions $\left\{a_{3}, b_{3}\right\}$ are defined similarly:

Definition 3.2 Given the functions $q(x, 0), q_{y}(x, 0)$, satisfying conditions (2.1), define the map

$$
\mathbb{S}_{3}:\left\{q(x, 0), q_{y}(x, 0)\right\} \rightarrow\left\{a_{3}(\lambda), b_{3}(\lambda)\right\}
$$

by

$$
\left(\begin{array}{l}
a_{3}(\lambda) \\
b_{3}(\lambda)
\end{array}\right)=\left[\Phi_{3}(0,0)\right]_{1}, \quad \lambda \in \mathbb{C}^{+},
$$

where $\left[\Phi_{3}(x, 0)\right]_{1}$ denotes the first column vector of the unique solution $\Phi_{3}(x, 0)$ of the Volterra linear integral equation

$$
\Phi(x, \lambda)=I-\int_{x}^{\infty} \mathrm{e}^{\Omega(\lambda)(\xi-x) \frac{\widehat{\frac{\widehat{\sigma}}{3}}}{2}} Q(\xi, 0, \lambda) \Phi(\xi, \lambda) d \xi
$$

and $Q(x, 0, \lambda)$ is given in terms of $q(x, 0)$ and $q_{y}(x, 0)$ by equation (2.5).

Proposition 3.2 The spectral functions $a_{3}(\lambda), b_{3}(\lambda)$ have the properties (i)-(v) of proposition (3.1), provided $a_{1}$ is replaced by $a_{3}, b_{1}$ is replaced by $b_{3}, \mathbb{S}_{1}$ is replaced by $\mathbb{S}_{3}$ and $L$ in replaced by 0 in all expressions.

The spectral functions at the $x=0$ boundary

Definition 3.3 Given the functions $q(0, y), q_{x}(0, y)$, satisfying conditions (2.1), define the map

$$
\mathbb{S}_{2}:\left\{q(0, y), q_{x}(0, y)\right\} \rightarrow\left\{a_{2}(\lambda), b_{2}(\lambda)\right\}
$$

by

$$
\left(\begin{array}{l}
a_{2}(\lambda) \\
b_{2}(\lambda)
\end{array}\right)=\left[\Phi_{2}(0,0)\right]_{1}, \quad \lambda \in \mathbb{C}^{+}
$$


where $\left[\Phi_{2}(0, y)\right]_{1}$ denotes the first column vector of the unique solution $\Phi_{2}(0, y)$ of the Volterra linear integral equation

$$
\Phi(y, \lambda)=I-i \int_{y}^{L} \mathrm{e}^{\omega(\lambda)(\eta-y) \widehat{\frac{\sigma_{3}}{2}}} Q(0, \eta,-\lambda) \Phi(\eta, \lambda) d \eta,
$$

and $Q(0, y, \lambda)$ is given in terms of $q(0, y)$ and $q_{x}(0, y)$ by equation (2.5).

Proposition 3.3 The spectral functions $a_{2}(\lambda), b_{2}(\lambda)$ have the following properties.

(i) $a_{2}(\lambda), b_{2}(\lambda)$ are analytic functions of $\lambda$, except for essential singularities at $\lambda=0$ and $\lambda=\infty$, bounded for $\operatorname{Re}(\lambda) \geq 0$.

(ii) $a_{2}(\lambda)=1+O\left(\frac{1}{\lambda}\right), b_{2}(\lambda)=O\left(\frac{1}{\lambda}\right)$ as $\lambda \rightarrow \infty, \operatorname{Re}(\lambda) \geq 0$.

(iii) $a_{2}(\lambda)=\cos \frac{q(0,0)}{2}+O(\lambda), b_{2}(\lambda)=i \sin \frac{q(0,0)}{2}+O(\lambda)$ as $\lambda \rightarrow 0, \operatorname{Re}(\lambda) \geq 0$.

(iv) $a_{2}(\lambda) a_{2}(-\lambda)-b_{2}(\lambda) b_{2}(-\lambda)=1, \lambda \in \mathbb{C}$.

(v) The map $\mathbb{Q}_{2}:\left\{a_{2}, b_{2}\right\} \rightarrow\left\{q(0, y) q_{y}(0, y)\right\}$, inverse to $\mathbb{S}_{2}$, is given by

$$
\begin{aligned}
\cos q(0, y) & =1-4 \lim _{\lambda \rightarrow \infty}\left(\lambda M_{y}\right)_{11}-2\left(\lim _{\lambda \rightarrow \infty}(\lambda M)_{21}\right)^{2} \\
q_{x}(0, y) & =i q_{y}(0, y)+2 \lim _{\lambda \rightarrow \infty}(\lambda M)_{21},
\end{aligned}
$$

where $M$ is the solution of the following Riemann-Hilbert problem:

- The function

$$
M(y, \lambda)= \begin{cases}M_{+}(y, \lambda) & \operatorname{Re} \lambda \geq 0 \\ M_{-}(y, \lambda) & \operatorname{Re} \lambda \leq 0\end{cases}
$$

is a sectionally meromorphic function of $\lambda \in \mathbb{C}$.

- $M=I+O\left(\frac{1}{\lambda}\right)$ as $\lambda \rightarrow \infty$, and

$$
M_{-}(y, \lambda)=M_{+}(y, \lambda) J_{2}(y, \lambda), \quad \lambda \in i \mathbb{R},
$$

where

$$
J_{2}(y, \lambda)=\left(\begin{array}{cc}
1 & -\frac{b_{2}(-\lambda)}{a_{2}(\lambda)} \mathrm{e}^{-\omega(\lambda) x} \\
\frac{b_{2}(\lambda)}{a_{2}(-\lambda)} \mathrm{e}^{\omega(\lambda) x} & \frac{1}{a_{2}(\lambda) a_{2}(-\lambda)}
\end{array}\right), \quad \lambda \in i \mathbb{R} .
$$

- $M$ satisfies appropriate residue conditions at the zeros of $a_{2}(\lambda)$.

\section{Proof of propositions (3.1)-(3.3)}

The proof of properties (i)-(iv) follows from the discussion in Section 2.2. In particular, property

(iii) follows from the asymptotic behaviour at $\lambda \rightarrow 0$, which can be derived by analysing equations (2.2)-(2.3) (see [41]), and is given by

$$
\Psi=\Psi_{0}+O(\lambda),|\lambda| \rightarrow 0, \quad \Psi_{0}(x, y)=\left(\begin{array}{cc}
\cos \frac{q(x, y)}{2} & i \sin \frac{q(x, y)}{2} \\
i \sin \frac{q(x, y)}{2} & \cos \frac{q(x, y)}{2}
\end{array}\right) .
$$


To prove (v), we note that the function $\Phi_{1}(x, \lambda)$ given by (2.17) is the unique solution of the ODE

$$
\begin{aligned}
& \Phi_{x}+\frac{\Omega(\lambda)}{2} \widehat{\sigma_{3}} \Phi=Q(x, L, \lambda) \Phi(x, \lambda), \\
& \lim _{x \rightarrow \infty} \Phi(x, \lambda)=I .
\end{aligned}
$$

Furthermore, $\Phi_{3}(x, \lambda)$ given by (2.19) is the solution of the same ODE problem, with $Q(x, L, \lambda)$ replaced by $Q(x, 0, \lambda)$.

Similarly, $\Phi_{2}(y, \lambda)$ given by $(2.18)$ is the unique solution of the ODE

$$
\begin{aligned}
& \Phi_{y}+\frac{\omega(\lambda)}{2} \widehat{\sigma_{3}} \Phi=i Q(0, y,-\lambda) \Phi(y, \lambda), \\
& \Phi(L, \lambda)=I .
\end{aligned}
$$

The spectral analysis of the above ODEs yields the desired result.

Regarding the rigorous derivation of the above results, we note the following: If $\left\{q(x, L), q_{y}(x, L)\right\}$, $\left\{q(x, 0), q_{y}(x, 0)\right\}$ and $\left\{q(y, 0), q_{x}(y, 0)\right\}$ are in $\mathbf{L}^{1}$, then the Volterra integral equations (3.1), (3.4) and (3.5) respectively, have a unique solution, and hence the spectral functions $\left\{a_{j}, b_{j}\right\}, j=1, . ., 3$, are well defined. Moreover, under the assumption (2.1) the spectral functions belong to $\mathbf{H}^{1}(\mathbb{R})$, hence the Riemann-Hilbert problems that determine the inverse maps can be characterized through the solutions of a Fredholm integral equation, see [10, 50].

\section{QED}

\subsection{The Riemann-Hilbert problem}

Theorem 3.1 Suppose that a subset of the boundary values $\left\{q(x, L), q_{y}(x, L)\right\},\left\{q(x, 0), q_{y}(x, 0)\right\}$, $0<x<\infty$, and $\left\{q(y, 0), q_{x}(y, 0)\right\}, 0<y<L$, satisfying (2.1), are prescribed as boundary conditions. Suppose that these prescribed boundary conditions are such that the global relations (2.22) can be used to characterize the remaining boundary values.

Define the spectral functions $\left\{a_{j}, b_{j}\right\}, j=1, . ., 3$, by definitions (3.1)-(3.3). Assume that the possible zeros $\left\{\lambda_{j}\right\}_{j=1}^{N_{1}}$ of $a_{1}(\lambda)$ and $\left\{\zeta_{j}\right\}_{j=1}^{N_{3}}$ of $a_{3}(\lambda)$ are as in assumption 2.29.

Define $M(x, y, \lambda)$ as the solution of the following $2 \times 2$ matrix Riemann-Hilbert problem:

- The function $M(x, y, \lambda)$ is a sectionally meromorphic function of $\lambda$ away from $\mathbb{R} \cup i \mathbb{R}$.

- The possible poles of the second column of $M$ occur at $\lambda=\zeta_{j}, j=1, \ldots, N_{3}$, in the first quadrant and at $\lambda=\lambda_{j}, j=1, \ldots, N_{1}$, in the second quadrant of the complex $\lambda$ plane.

The possible poles of the first column of $M$ occur at $\lambda=-\lambda_{j}\left(j=1, \ldots, N_{1}\right)$ and $\lambda=-\zeta_{j}$ $\left(j=1, \ldots, N_{3}\right)$.

The associated residue conditions satisfy the relations (2.32).

- $M=I+O\left(\frac{1}{\lambda}\right)$ as $\lambda \rightarrow \infty$, and

$$
M_{-}(x, y, \lambda)=M_{+}(x, y, \lambda) J(x, y, \lambda), \quad \lambda \in \mathbb{R} \cup i \mathbb{R},
$$

where $M=M_{+}$for $\lambda$ in the first or third quadrant, and $M=M_{-}$for $\lambda$ in the second or fourth quadrant of the complex $\lambda$ plane, and $J$ is defined in terms of $\left\{a_{j}, b_{j}\right\}$ by equations (2.26). 
Then $M$ exists and is unique, provided that the $\mathbf{H}^{1}$ norm of of the spectral functions is sufficiently small.

Define $q(x, y)$ is terms of $M(x, y, \lambda)$ by

$$
\begin{aligned}
q_{x}-i q_{y} & =2 \lim _{\lambda \rightarrow \infty}(\lambda M)_{21}, \\
\cos q(x, y) & =1+4 i\left(\lim _{\lambda \rightarrow \infty}\left(\lambda M_{x}\right)_{11}\right)+2\left(\lim _{\lambda \rightarrow \infty}(\lambda M)_{21}\right)^{2} .
\end{aligned}
$$

Then $q(x, y)$ solves (1.1). Furthermore, $q(x, y)$ evaluated at the boundary, yields the functions used for the computation of the spectral functions.

Proof: Under the assumptions (2.1), the spectral functions are in $\mathbf{H}^{1}$.

In the case when $a_{1}(\lambda)$ and $a_{3}(\lambda)$ have no zeros, the Riemann-Hilbert problem is regular and it is equivalent to a Fredholm integral equation. However, we have not been able to establish a vanishing lemma, hence we require a small norm assumption for solvability.

If $a_{1}(\lambda)$ and $a_{3}(\lambda)$ have zeros, the singular RH problem can be mapped to a regular one coupled with a system of algebraic equations [21]. Moreover, it follows from standard arguments, using the dressing method [48,49], that if $M$ solves the above $\mathrm{RH}$ problem and $q(x, y)$ is defined by (3.7)-(3.8), then $q(x, y)$ solves equation (1.1). The proof that $q$ evaluated at the boundary yields the functions used for the computation of the spectral functions follows arguments similar to the ones used in [23].

QED

\section{Linearizable boundary conditions}

We now concentrate on the particular boundary conditions (1.3). We note that these boundary conditions are symmetric with respect to the line $y=\frac{L}{2}$. Hence, if $q(x, y)$ is a solution, so is $q(x, L-y)$. Assuming that the solution is unique, we can conclude that

$$
q(x, y)=q(x, L-y), \quad 0<x<\infty, 0<y<L .
$$

These boundary conditions are not compatible at the corners of the domain, and therefore introduce a discontinuity at each corner. It turns out that these discontinuities imply that if $q(x, y)$ is the solution of the resulting boundary value problem, then the function $q_{y}(x, 0)=-q_{y}(x, L)$ is not integrable near $x=0$. Similarly, $q_{x}(0, y)$ is not integrable near $y=0$ and $y=L$. Hence we cannot guarantee that the results of propositions 3.1-3.3 hold. In particular, the spectral functions as given by (2.17)-(2.19) and the resulting Riemann-Hilbert problem are not well defined.

To overcome this lack of regularity, we will employ a gauge transformation to define a modified Lax pair. This transformation is motivated by the recent analysis of the linearised problem [2], which we summarize in Appendix A. For the linear case, it can be shown that the behaviour of the boundary function $q_{y}(x, 0)$ as $x \rightarrow 0$ is given by $q_{y}(x, 0) \sim \frac{2 d}{\pi x}$. The contribution of this term can be eliminated by an appropriate gauge transformation. The advantage of the new Lax pair we define below by adapting the linear gauge transformation to the nonlinear setting, is that the spectral functions and the Riemann-Hilbert problem are well defined, indicating that in the nonlinear case, as in the linear, the singular behaviour introduced by the terms $q_{y}(x, 0)$ and $q_{x}(0, y)$ is eliminated. 


\subsection{A new Lax pair}

The linearised version of the elliptic sine-Gordon equation, namely the modified Helmholtz equation, with the boundary conditions (1.3), is discussed in Appendix A, where we show how, by incorporating appropriately in the differential form associated with the linear equation the term

$$
\kappa(x, y)=-\frac{1}{4} \int_{x}^{\infty} q_{y}(\xi, y) d \xi
$$

the spectral problem is regularized.

Motivated by the linear analysis, we now introduce a new eigenfunction $\Phi$ via the gauge transformation

$$
g \Phi=\Psi, \quad g(x, y):=e^{\kappa(x, y) \sigma_{1}}=\left(\begin{array}{cc}
\cosh \kappa(x, y) & \sinh \kappa(x, y) \\
\sinh \kappa(x, y) & \cosh \kappa(x, y)
\end{array}\right)
$$

where $\Psi$ denotes the solution of the Lax pair (2.2)-(2.3) and $\kappa(x, y)$ is given by (4.2). Note that

$$
\kappa_{x}(x, y)=\frac{1}{4} q_{y}(x, y), \quad \kappa_{y}(x, y)=-\frac{1}{4} \int_{x}^{\infty} \sin q(\xi, y) d \xi-\frac{1}{4} q_{x}(x, y) .
$$

The transformation matrix $g(x, y)$ has unit determinant, and is chosen to satisfy the property

$$
g^{-1} g_{x}=g_{x} g^{-1}=\frac{1}{4}\left(\begin{array}{cc}
0 & q_{y}(x, y) \\
q_{y}(x, y) & 0
\end{array}\right)
$$

Let the function $\Phi$ satisfy the Lax pair

$$
\begin{aligned}
& \Phi_{x}+\frac{\Omega(\lambda)}{2}\left[\sigma_{3}, \Phi\right]=V_{1}(x, y, \lambda) \Phi, \\
& \Phi_{y}+\frac{\omega(\lambda)}{2}\left[\sigma_{3}, \Phi\right]=V_{2}(x, y, \lambda) \Phi,
\end{aligned}
$$

with

$$
\begin{gathered}
V_{1}(x, y, \lambda)=-g^{-1} g_{x}+g^{-1} Q(x, y, \lambda) g-\frac{\Omega(\lambda)}{2} g^{-1}\left[\sigma_{3}, g\right] \\
=i\left(\begin{array}{cc}
\frac{\cosh (2 \kappa) \lambda^{2}-\lambda^{2}-\cosh (2 \kappa-i q)+1}{4 \lambda} & \frac{\sinh (2 \kappa) \lambda^{2}+q_{x} \lambda-\sinh (2 \kappa-i q)}{4 \lambda} \\
\frac{-\sinh (2 \kappa) \lambda^{2}+q_{x} \lambda+\sinh (2 \kappa-i q)}{4 \lambda} & \frac{-\cosh (2 \kappa) \lambda^{2}+\lambda^{2}+\cosh (2 \kappa-i q)-1}{4 \lambda}
\end{array}\right), \\
V_{2}(x, y, \lambda)=-g^{-1} g_{y}+g^{-1} i Q(x, y,-\lambda) g-\frac{\omega(\lambda)}{2} g^{-1}\left[\sigma_{3}, g\right] \\
=\left(\begin{array}{cc}
-\frac{\cosh (2 \kappa) \lambda^{2}-\lambda^{2}+\cosh (2 \kappa-i q)-1}{4 \lambda} & -\frac{\sinh (2 \kappa) \lambda^{2}-\left(\int_{x}^{\infty} \sin q d \xi+i q_{y}\right) \lambda+\sinh (2 \kappa-i q)}{4 \lambda} \\
\frac{\sinh (2 \kappa) \lambda^{2}+\left(\int_{x}^{\infty} \sin q d \xi+i q_{y}\right) \lambda+\sinh (2 \kappa-i q)}{4 \lambda} & \frac{\cosh (2 \kappa) \lambda^{2}-\lambda^{2}+\cosh (2 \kappa-i q)-1}{4 \lambda}
\end{array}\right) .
\end{gathered}
$$

We now use the eigenfunctions determined by the Lax pair (4.4)-(4.5) to define new spectral functions. Namely, in analogy with (2.17)-(2.19), we define

$$
S_{1}(\lambda)=\varphi_{1}(0, \lambda), \quad \varphi_{1}(x, \lambda)=I-\int_{x}^{\infty} \mathrm{e}^{\Omega(\lambda)(\xi-x) \frac{\widehat{\frac{\sigma}{3}}}{2}} V_{1}(\xi, L, \lambda) \varphi_{1}(\xi, \lambda) d \xi
$$




$$
\begin{array}{ll}
\lambda \in\left(\mathbb{C}^{+}, \mathbb{C}^{-}\right), \quad 0<x<\infty, \\
S_{2}(\lambda)=\varphi_{2}(0, \lambda), \quad \varphi_{2}(y, \lambda)=I-\int_{y}^{L} \mathrm{e}^{\omega(\lambda)(\eta-y) \frac{\widehat{\sigma_{3}}}{2}} V_{2}(0, \eta, \lambda) \varphi_{2}(\eta, \lambda) d \eta, \\
\lambda \in \mathbb{C}, \quad 0<y<L, \\
S_{3}(\lambda)=\varphi_{3}(0, \lambda), \quad \varphi_{3}(x, \lambda)=I-\int_{x}^{\infty} \mathrm{e}^{\Omega(\lambda)(\xi-x) \frac{\widehat{\widehat{\sigma}}}{2}} V_{1}(\xi, 0, \lambda) \varphi_{3}(\xi, \lambda) d \xi, \\
\lambda \in\left(\mathbb{C}^{+}, \mathbb{C}^{-}\right), \quad 0<x<\infty .
\end{array}
$$

Note that $V_{1}$ does not involve $q_{y}(x, y)$, and $V_{2}$ does not involve $q_{x}(x, y)$, the terms respectively responsible, at least in the linear case, for the non-integrable behaviour.

Note also that, since the symmetry relation (2.20) holds for $V_{1}, V_{2}$ in place of $Q$, we can represent the matrices $\varphi_{i}$ in the form

$$
\begin{aligned}
\varphi_{1} & =\left(\begin{array}{ll}
A_{1}(x, \lambda) & B_{1}(x,-\lambda) \\
B_{1}(x, \lambda) & A_{1}(x,-\lambda)
\end{array}\right), \quad \varphi_{2}=\left(\begin{array}{ll}
A_{2}(y, \lambda) & B_{2}(y,-\lambda) \\
B_{2}(y, \lambda) & A_{2}(y,-\lambda)
\end{array}\right), \\
\varphi_{3} & =\left(\begin{array}{ll}
A_{3}(x, \lambda) & B_{3}(x,-\lambda) \\
B_{3}(x, \lambda) & A_{3}(x,-\lambda)
\end{array}\right),
\end{aligned}
$$

and set

$$
S_{i}(\lambda)=\left(\begin{array}{cc}
a_{i}(\lambda) & b_{i}(-\lambda) \\
b_{i}(\lambda) & a_{i}(-\lambda)
\end{array}\right), \quad i=1,2,3
$$

The rest of the general construction of sections 2.3-3.2 is formally valid with the spectral functions $a_{j}(\lambda), b_{j}(\lambda), j=1,2,3$ as defined by (4.12), except for the statement (i)-(iii) and (v) of propositions 3.1-3.3.

\section{Symmetry conditions}

Given the boundary conditions (1.3), equations (4.8)-(4.10) are written explicitly as follows:

$$
\begin{aligned}
& \varphi_{1}(x, \lambda)=I-\frac{\Omega(\lambda)}{2} \int_{x}^{\infty} \mathrm{e}^{\Omega(\lambda)(\xi-x) \frac{\widehat{\sigma_{3}}}{2}}\left(\begin{array}{cc}
1-\cosh 2 \kappa & -\sinh 2 \kappa \\
\sinh 2 \kappa & \cosh 2 \kappa-1
\end{array}\right)(\xi, L) \varphi_{1}(\xi, \lambda) d \xi, \\
& 0<x<\infty, \quad \lambda \in\left(\mathbb{C}^{+}, \mathbb{C}^{-}\right), \\
& \varphi_{2}(y, \lambda)=I-\int_{y}^{L} \mathrm{e}^{\omega(\lambda)(\eta-y) \frac{\widehat{\sigma}}{2}} \\
& \left(\begin{array}{cc}
-\frac{\cosh (2 \kappa) \lambda^{2}-\lambda^{2}+\cosh (2 \kappa-i d)-1}{4 \lambda} & \frac{-\sinh (2 \kappa) \lambda^{2}+\lambda \int_{x}^{\infty} \sin q d \xi-\sinh (2 \kappa-i d)}{4 \lambda} \\
\frac{\sinh (2 \kappa) \lambda^{2}+\lambda \int_{x}^{\infty} \sin q d \xi+\sinh (2 \kappa-i d)}{4 \lambda} & \frac{\cosh (2 \kappa) \lambda^{2}-\lambda^{2}+\cosh (2 \kappa-i d)-1}{4 \lambda}
\end{array}\right)(0, \eta) \varphi_{2}(\eta, \lambda) d \eta, \\
& 0<y<L, \quad \lambda \in \mathbb{C}, \\
& \varphi_{3}(x, \lambda)=I-\frac{\Omega(\lambda)}{2} \int_{x}^{\infty} \mathrm{e}^{\Omega(\lambda)(\xi-x) \frac{\widehat{\sigma_{3}}}{2}}\left(\begin{array}{cc}
1-\cosh 2 \kappa & -\sinh 2 \kappa \\
\sinh 2 \kappa & \cosh 2 \kappa-1
\end{array}\right)(\xi, 0) \varphi_{3}(\xi, \lambda) d \xi, \\
& 0<x<\infty, \quad \lambda \in\left(\mathbb{C}^{+}, \mathbb{C}^{-}\right) .
\end{aligned}
$$


Using that $\kappa(x, 0)=-\kappa(x, L)$, we can immediately conclude that

$$
\left(\begin{array}{c}
A_{1}(x, \lambda) \\
B_{1}(x, \lambda)
\end{array}\right)=\left(\begin{array}{c}
A_{3}(x, \lambda) \\
-B_{3}(x, \lambda)
\end{array}\right) \Longrightarrow \quad a_{1}(\lambda)=a_{3}(\lambda), \quad b_{1}(\lambda)=-b_{3}(\lambda)
$$

where $A_{i}, B_{1}, a_{i}, b_{i}$ are as in (4.11)-(4.12).

In equations (4.13) and (4.15), the only dependence on $\lambda$ is through $\Omega(\lambda)$. Thus, since $\Omega\left(-\frac{1}{\lambda}\right)=$ $\Omega(\lambda)$, it follows that the vector functions $\left(A_{1}, B_{1}\right)$ and $\left(A_{3}, B_{3}\right)$ satisfy the same symmetry properties. Hence,

$$
a_{j}\left(-\frac{1}{\lambda}\right)=a_{j}(\lambda), \quad b_{j}\left(-\frac{1}{\lambda}\right)=b_{j}(\lambda), \quad j=1,3, \quad \operatorname{Im}(\lambda) \geq 0 .
$$

It turns out that the vector function $\left(A_{2}, B_{2}\right)$ also satisfies a certain symmetry condition, as stated in the following proposition.

Proposition 4.1 Let $q_{x}(0, y)$ be a sufficiently smooth function. Then the vector solution of the linear Volterra integral equation (4.14) satisfies the following symmetry conditions:

$$
\begin{gathered}
A_{2}\left(y, \frac{1}{\lambda}\right)=\frac{A_{2}(y, \lambda)-F(\lambda) B_{2}(y, \lambda)+F(\lambda) \mathrm{e}^{\omega(\lambda)(y-L)} B_{2}(y,-\lambda)-F(\lambda)^{2} \mathrm{e}^{\omega(\lambda)(y-L)} A_{2}(y,-\lambda)}{1-F(\lambda)^{2}} \\
B_{2}\left(y, \frac{1}{\lambda}\right)=\frac{B_{2}(y, \lambda)-F(\lambda) A_{2}(y, \lambda)+F(\lambda) \mathrm{e}^{\omega(\lambda)(y-L)} A_{2}(y,-\lambda)-F(\lambda)^{2} \mathrm{e}^{\omega(\lambda)(y-L)} B_{2}(y,-\lambda)}{1-F(\lambda)^{2}} \\
0<y<L, \quad \lambda \in \mathbb{C}
\end{gathered}
$$

where the function $F(\lambda)$ is defined by

$$
F(\lambda)=i \frac{1-\lambda^{2}}{1+\lambda^{2}} \tan \frac{d}{2}
$$

Proof: Define a function $\phi_{2}(y, \lambda)$ by

$$
\varphi_{2}(y, \lambda)=\phi_{2}(y, \lambda) \mathrm{e}^{\frac{\omega(\lambda)}{2} \sigma_{3}(y-L)}
$$

where $\varphi_{2}$ is defined by (4.14). It follows that $\phi_{2}$ satisfies the ODE

$$
\begin{aligned}
& \left(\phi_{2}\right)_{y}=V \phi_{2}, \\
& \phi_{2}(L, \lambda)=I, \quad 0<y<L,
\end{aligned}
$$

where

$$
V(y, \lambda)=V_{2}(0, y, \lambda)-\frac{\omega(0, y, \lambda)}{2} \sigma_{3} .
$$

We seek a nonsingular matrix $R(\lambda)$, independent of $y$, such that

$$
V\left(y, \frac{1}{\lambda}\right)=R(\lambda) V(y, \lambda) R(\lambda)^{-1} .
$$


It can be verified that such a matrix is given by

$$
R(\lambda)=\left(\begin{array}{cc}
1 & -F(\lambda) \\
-F(\lambda) & 1
\end{array}\right)
$$

where $F$ is defined by (4.19).

Replacing in equation (4.21) $\lambda$ by $\frac{1}{\lambda}$, and using (4.23), we find the following equation:

$$
\left(R(\lambda)^{-1} \phi_{2}\left(y, \frac{1}{\lambda}\right)\right)_{y}=V(y, \lambda)\left(R(\lambda)^{-1} \phi_{2}\left(y, \frac{1}{\lambda}\right)\right)
$$

hence

$$
R(\lambda)^{-1} \phi_{2}\left(y, \frac{1}{\lambda}\right)=\phi_{2}(y, \lambda) C(\lambda)
$$

where $C$ is a $y$-independent matrix. Using the second of equations (4.21), it follows that $C=R^{-1}$, and therefore

$$
\phi_{2}\left(y, \frac{1}{\lambda}\right)=R(\lambda) \phi_{2}(y, \lambda) R(\lambda)^{-1} .
$$

This equation and equation (4.20) imply

$$
\varphi_{2}\left(y, \frac{1}{\lambda}\right)=R(\lambda) \varphi_{2}(y, \lambda)\left(\mathrm{e}^{-\omega(\lambda) \frac{\widehat{\sigma_{3}}}{2}(y-L)} R(\lambda)^{-1}\right) .
$$

The first column vector of this equation implies (4.18).

\section{QED}

Remark 4.1 Recalling that $a_{2}(\lambda)=A_{2}(0, \lambda)$, and $b_{2}(\lambda)=B_{2}(0, \lambda)$, equations (4.18) immediately imply the following important relations:

$$
\begin{aligned}
& a_{2}\left(\frac{1}{\lambda}\right)=\frac{a_{2}(\lambda)-F(\lambda) b_{2}(\lambda)+F(\lambda) \mathrm{e}^{-\omega(\lambda) L} b_{2}(-\lambda)-F(\lambda)^{2} \mathrm{e}^{-\omega(\lambda) L} a_{2}(-\lambda)}{1-F(\lambda)^{2}}, \\
& b_{2}\left(\frac{1}{\lambda}\right)=\frac{b_{2}(\lambda)-F(\lambda) a_{2}(\lambda)+F(\lambda) \mathrm{e}^{-\omega(\lambda) L} a_{2}(-\lambda)-F(\lambda)^{2} \mathrm{e}^{-\omega(\lambda) L} b_{2}(-\lambda)}{1-F(\lambda)^{2}}, \quad \lambda \in \mathbb{C} \backslash\{0\} .
\end{aligned}
$$

In summary, the basic equations characterizing the spectral functions are:

(a) the symmetry relations (4.16), (4.17) and (4.26);

(b) the global relations (2.22);

(c) the conditions of unit determinant.

In the next lemma, we collect some important consequences of these conditions. For simplicity, we will use the notations

$$
f:=f(\lambda), \quad \hat{f}:=f(-\lambda) .
$$


Lemma 4.1 The spectral functions satisfy the following relations:

$$
\begin{array}{ccc}
a_{3} \hat{a}_{3}-b_{3} \hat{b}_{3}=1, & \lambda \in \mathbb{R}, \\
\hat{a}_{3} b_{3}-a_{3} \hat{b}_{3}=G, & \lambda \in \mathbb{R}, \\
b_{2}=\left(1+\mathrm{e}^{-\omega(\lambda) L}\right) \hat{a}_{3} b_{3}, & \lambda \in \mathbb{R},
\end{array}
$$

where the function $G(\lambda)$ is defined by

$$
G(\lambda):=F(\lambda) \tanh \left(\frac{L \omega}{2}\right)=i \tan \left(\frac{d}{2}\right) \frac{1-\lambda^{2}}{1+\lambda^{2}} \frac{e^{\omega(\lambda) L}-1}{e^{\omega(\lambda) L}+1} .
$$

Proof: Equation (4.27) is just the condition of unit determinant.

Using the symmetry condition (4.16) to eliminate $a_{1}$ and $b_{1}$ from the global relations (2.22), we find

$$
\begin{array}{cl}
a_{3}\left[\hat{a}_{2}-1\right]=\hat{b}_{2} b_{3}, & \lambda \in \mathbb{R}, \\
b_{3}\left[\mathrm{e}^{-\omega(\lambda) L}+a_{2}\right]=a_{3} b_{2}, & \lambda \in \mathbb{R} .
\end{array}
$$

The equations (4.31) together with the equations obtained by letting $\lambda \rightarrow-\lambda$ in (4.31) are four equations which can be solved for the four functions $\left\{a_{2}, b_{2}, \hat{a}_{2}, \hat{b}_{2}\right\}$ with the result that

$$
\begin{array}{ll}
a_{2}=\left(1+\mathrm{e}^{-\omega(\lambda) L}\right) a_{3} \hat{a}_{3}-e^{-\omega(\lambda) L}, & \lambda \in \mathbb{R}, \\
b_{2}=\left(1+\mathrm{e}^{-\omega(\lambda) L}\right) \hat{a}_{3} b_{3}, & \lambda \in \mathbb{R} .
\end{array}
$$

This proves (4.29)

Replacing $\lambda$ by $-1 / \lambda$ in (4.31) and using the symmetry (4.17), we find

$$
\begin{array}{ccrl}
a_{3}(\lambda)\left[a_{2}\left(\frac{1}{\lambda}\right)-1\right]=b_{2}\left(\frac{1}{\lambda}\right) b_{3}(\lambda), & \lambda \in \mathbb{R}, \\
b_{3}(\lambda)\left[\mathrm{e}^{\omega(\lambda) L}+a_{2}\left(-\frac{1}{\lambda}\right)\right]=a_{3}(\lambda) b_{2}\left(-\frac{1}{\lambda}\right), & \lambda \in \mathbb{R} .
\end{array}
$$

Consider the two equations (4.33) together with the two equations obtained by letting $\lambda \rightarrow-\lambda$ in (4.33). We can eliminate $a_{2}\left( \pm \frac{1}{\lambda}\right)$ and $b_{2}\left( \pm \frac{1}{\lambda}\right)$ from these four equations by using the symmetry relations (4.26) as well as the symmetry relations obtained by letting $\lambda \rightarrow-\lambda$ in (4.26). The resulting four equations can then be solved for the four functions $\left\{a_{2}, b_{2}, \hat{a}_{2}, \hat{b}_{2}\right\}$ with the result that

$$
\begin{aligned}
& a_{2}=\frac{\left(a_{3} F+b_{3}\right)\left(\hat{a}_{3} F-\hat{b}_{3}\right) e^{-L \omega}-\left(a_{3}-b_{3} F\right)\left(\hat{a}_{3}+\hat{b}_{3} F\right)}{F^{2}-1}, \\
& b_{2}=\frac{\left(a_{3}+b_{3} F\right)\left(\hat{a}_{3} F-\hat{b}_{3}\right) e^{-L \omega}-\left(a_{3}-b_{3} F\right)\left(\hat{a}_{3} F+\hat{b}_{3}\right)}{F^{2}-1} .
\end{aligned}
$$

Comparing (4.32a) with (4.34a), we find (4.28). QED

The functions $a_{3}(\lambda)$ and $b_{3}(\lambda)$ are defined by (4.12) only for $\lambda$ in the upper half-plane. However, equation (4.29) implies that $a_{3}(\lambda)$ and $b_{3}(\lambda)$ can be analytically extended to the whole complex plane. Indeed, equation (4.29) provides the analytic continuation of $a_{3}(\lambda)$ into $\mathbb{C}^{-}$:

$$
a_{3}(-\lambda)=\frac{b_{2}(\lambda)}{b_{3}(\lambda)\left[1+e^{-\omega(\lambda) L}\right]}, \quad \lambda \in \mathbb{C}^{+} .
$$


Similarly, equation (4.29) with $\lambda$ replaced with $-\lambda$ provides the analytic continuation of $b_{3}(\lambda)$ into $\mathbb{C}^{-}$:

$$
b_{3}(-\lambda)=\frac{b_{2}(-\lambda)}{a_{3}(\lambda)\left[1+e^{\omega(\lambda) L}\right]}, \quad \lambda \in \mathbb{C}^{+} .
$$

Adopting these extended definitions of $a_{3}(\lambda)$ and $b_{3}(\lambda)$, analytic continuation implies that the relations (4.27)-(4.29) and the global relations (4.31) are valid in the whole complex plane.

Proposition 4.2 The spectral functions satisfy the equations

$$
\begin{array}{cc}
a_{3} \hat{a}_{3}-b_{3} \hat{b}_{3}=1, & \lambda \in \mathbb{C}, \\
\hat{a}_{3} b_{3}-a_{3} \hat{b}_{3}=G, & \lambda \in \mathbb{C}, \\
b_{2}=\left(1+\mathrm{e}^{-\omega(\lambda) L}\right) \hat{a}_{3} b_{3}, & \lambda \in \mathbb{C},
\end{array}
$$

as well as the global relations

$$
\begin{array}{cc}
a_{3}\left[\hat{a}_{2}-1\right]=\hat{b}_{2} b_{3}, & \lambda \in \mathbb{C}, \\
b_{3}\left[\mathrm{e}^{-\omega(\lambda) L}+a_{2}\right]=a_{3} b_{2}, & \lambda \in \mathbb{C},
\end{array}
$$

where the known function $G(\lambda)$ is given by (4.30).

\section{Spectral theory in the linearisable case}

In appendix $A$ we give the solution of the linear case. In this case, the dependence on the unknown spectral function $B(\lambda)$ is resolved by mapping the basic Riemann-Hilbert problem to an equivalent but simpler one. To define this mapping, in the linear case, it is convenient to employ the two equations (A.19) and (A.20).

For the nonlinear problem, we will use the following equations, which provide the nonlinear analogues of equations (A.19) and (A.20):

$$
\frac{b_{3}(\lambda)}{a_{3}(\lambda) h(\lambda)}-\frac{b_{3}(-\lambda)}{a_{3}(\lambda)}=\frac{G(\lambda)}{h(\lambda)}, \quad \lambda \in \mathbb{C},
$$

and

$$
\frac{b_{2}(\lambda)}{a_{3}(-\lambda)^{2}}-\left(1+\mathrm{e}^{-\omega(\lambda) L}\right) \frac{b_{3}(-\lambda)}{a_{3}(-\lambda) h(-\lambda)}=\left(1+\mathrm{e}^{-\omega(\lambda) L}\right) \frac{G(\lambda)}{h(-\lambda)}, \quad \lambda \in \mathbb{C},
$$

where the unknown function $h(\lambda)$ is defined by

$$
h(\lambda)=a_{3}(\lambda)^{2}-b_{3}(\lambda)^{2}, \quad \lambda \in \mathbb{C},
$$

and the known function $G(\lambda)$ is defined by (4.30). Note that from the above properties it follows that $\frac{b_{3}(-\lambda)}{a_{3}(\lambda)}$ is well defined at the zeros of the function $h(\lambda)$. Moreover, in the linear limit,

$$
b_{3}(\lambda) \rightarrow B_{3}(\lambda), \quad a_{3}(\lambda) \rightarrow 1, \quad h(\lambda) \rightarrow 1, \quad \tan \frac{d}{2} \rightarrow \frac{d}{2},
$$

and equations (5.1) and (5.2) become equations (A.19) and (A.20). 
Equation (5.1) and (5.2) are a direct consequence of (4.37)-(4.39). Indeed,

$$
\begin{aligned}
a_{3}(\lambda) G(\lambda) & =a_{3}(\lambda)\left[a_{3}(-\lambda) b_{3}(\lambda)-a_{3}(\lambda) b_{3}(-\lambda)\right] \\
& =-a_{3}(\lambda)^{2} b_{3}(-\lambda)+b_{3}(\lambda)\left[1+b_{3}(\lambda) b_{3}(-\lambda)\right] \\
& =b_{3}(\lambda)-\left[a_{3}(\lambda)^{2}-b_{3}(\lambda)^{2}\right] b_{3}(-\lambda),
\end{aligned}
$$

which is equivalent to equation (5.1) (in the first and second equations above we have used equations (4.38) and (4.37) respectively). Furthermore,

$$
\frac{b_{2}(\lambda)}{a_{3}(-\lambda)^{2}}=\left(1+\mathrm{e}^{-\omega(\lambda) L}\right) \frac{b_{3}(\lambda)}{a_{3}(-\lambda)}=\left(1+\mathrm{e}^{-\omega(\lambda) L}\right)\left[\frac{b_{3}(-\lambda)}{a_{3}(-\lambda) h(-\lambda)}-\frac{G(-\lambda)}{h(-\lambda)}\right],
$$

which, using $G(-\lambda)=-G(\lambda)$, becomes equation (5.2) (in the first and second equations above we have used the relation (4.39) and equation (5.1) with $\lambda$ replaced by $-\lambda$ ).

\section{The properties of the functions $G(\lambda)$ and $h(\lambda)$}

We give a summary of some of the properties of the function $G(\lambda)$ given by (4.30) and of the unknown function $h$ given by (5.3).

The set of poles of $G(\lambda)$, denoted by $P_{G} \subset \mathbb{C}$, is given by

$$
\begin{aligned}
P_{G} & =\left\{\lambda \mid e^{\omega(\lambda) L}+1=0\right\} \\
& =\left\{\lambda_{n}=\frac{i}{L}\left(\pi(2 n+1) \pm \sqrt{L^{2}+(2 n+1)^{2} \pi^{2}}\right) \mid n \in \mathbb{Z}\right\} .
\end{aligned}
$$

Note that $G(\lambda)$ has no poles at $\lambda= \pm i$ since the term $e^{\omega L}-1$ vanishes at these points.

Proposition 5.1 The following statements hold:

(a) $G(\lambda)$ admits the symmetries $G(\lambda)=-G(-\lambda)=-G(1 / \lambda), \lambda \in \mathbb{C}$.

(b) $G(\lambda)$ has essential singularities at $\infty$ and at 0 and a countable number of simple poles on the imaginary axis accumulating at $\pm i \infty$ and at $0 . G(\lambda)$ has no other singularities.

(c) Each of the functions $1 \pm G(\lambda)$ has a countable number of zeros. All these zeros lie on the imaginary axis and they accumulate only at $\pm i \infty$ and at 0.

(d) The set of zeros of the function $1-G(\lambda)$ is the disjoint union of the set of zeros of $a_{3}(\lambda)-b_{3}(\lambda)$ and the set of zeros of $a_{3}(-\lambda)+b_{3}(-\lambda)$.

(e) The set of zeros of the function $1+G(\lambda)$ is the disjoint union of the set of zeros of $a_{3}(\lambda)+b_{3}(\lambda)$ and the set of zeros of $a_{3}(-\lambda)+b_{3}(-\lambda)$.

$(f)$ The set of zeros of the function $1-G^{2}(\lambda)$ is the disjoint union of the set of zeros of $h(\lambda)$ and the set of zeros of $h(-\lambda)$.

(g) The function $h(\lambda)$ has a double pole at each point in the set $P_{G} \cap \mathbb{C}^{-}$. The function $h(-\lambda)$ has a double pole at each point in the set $P_{G} \cap \mathbb{C}^{+}$. The functions $h(\lambda)$ and $h(-\lambda)$ do not have any other poles. 


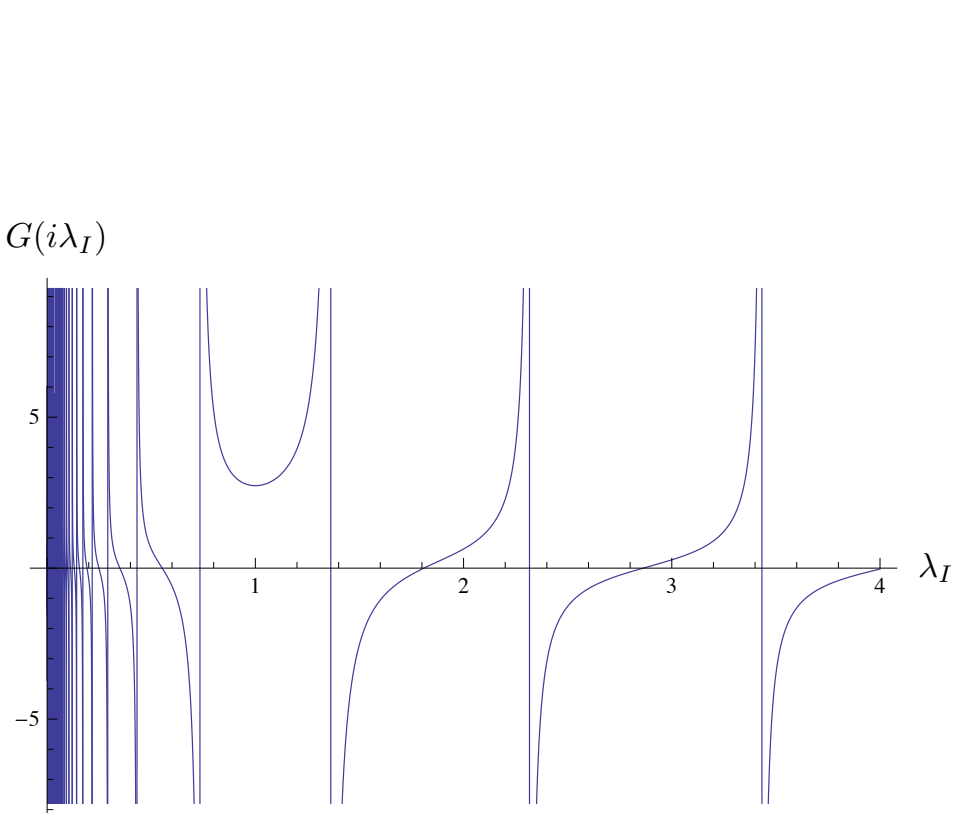

(a)



(b)

Figure 4: (a) The graph of $G(\lambda)$ for $\lambda$ on the imaginary axis when $L=10$ and $d=1$. (b) The corresponding picture of the poles of $G(\lambda)$ and the zeros of $G(\lambda) \pm 1$. The poles and zeros accumulate at the origin and at infinity.

Proof: The proof of ( $a$ ) follows from the definition (4.30). The proof of (b) follows from the same definition and from equation (5.4).

In order to prove $(c)$, we note that the function $G$ is purely real for $\lambda \in i \mathbb{R}$ :

$$
G\left(i \lambda_{I}\right)=-\frac{1+\lambda_{I}^{2}}{1-\lambda_{I}^{2}} \tan \left(\frac{d}{2}\right) \tan \left(L \frac{\lambda_{I}^{2}-1}{4 \lambda_{I}}\right), \quad \lambda_{I} \in \mathbb{R} .
$$

As $\lambda_{I}$ increases from 0 to $+\infty$, the argument $L \frac{\lambda_{I}^{2}-1}{4 \lambda_{I}}$ increases from $-\infty$ to $\infty$. It follows that each of the functions $1+G(\lambda)$ and $1-G(\lambda)$ has an infinite number of zeros on the imaginary axis, see figure 4. More precisely, if $i p_{1}$ and $i p_{2}$ are two consecutive poles of $G$ on the positive imaginary axis, then, unless $p_{1}<1<p_{2}$, there is exactly one zero of $1-G(\lambda)$ and one zero of $1+G(\lambda)$ belonging to the interval $\left(i p_{1}, i p_{2}\right)$. If $p_{1}<1<p_{2}$, then there are no zeros of $1+G(\lambda)$ in the interval $\left(i p_{1}, i p_{2}\right)$ and there are two (counted with multiplicity) or no zeros of $1-G(\lambda)$ in this interval depending on whether $G(i)=\frac{L}{2} \tan (d / 2)$ is $\leq 1$ or $>1$. Since the poles of $G(\lambda)$ accumulate at 0 and at $\pm i \infty$, the same is true for the zeros of $1 \pm G(\lambda)$. This proves $(c)$. Taking the sum and difference of (4.37) and (4.38) we find

$$
\left(a_{3} \pm b_{3}\right)\left(\hat{a}_{3} \mp \hat{b}_{3}\right)=1 \pm G, \quad \lambda \in \mathbb{C} .
$$

It follows that

$$
h(\lambda) h(-\lambda)=1-G^{2}(\lambda), \quad \lambda \in \mathbb{C} .
$$


Equation (5.5) implies that either $a_{3}-b_{3}$ or $\hat{a}_{3}+\hat{b}_{3}$ vanishes whenever $1-G=0$. Since all zeros of $1-G$ are simple, the functions $a_{3}-b_{3}$ and $\hat{a}_{3}+\hat{b}_{3}$ cannot simultaneously vanish at one of these zeros. In order to prove $(d)$, it only remains to show that $1-G$ vanishes whenever $a_{3}-b_{3}$ or $\hat{a}_{3}+\hat{b}_{3}$ does. Equation (5.5) suggests that this is true; however, it is conceivable that a zero (pole) of $a_{3}-b_{3}$ could coincide with a pole (zero) of $\hat{a}_{3}+\hat{b}_{3}$ in such a way that the product $\left(a_{3}-b_{3}\right)\left(\hat{a}_{3}+\hat{b}_{3}\right)=1-G$ remains nonzero. We show now that this cannot occur.

Suppose $\lambda_{0}$ is a zero of $a_{3}(\lambda)-b_{3}(\lambda)$. The unit determinant condition (4.37) implies that $a_{3}(\lambda)$ and $b_{3}(\lambda)$ cannot simultaneously vanish. Thus,

$$
a_{3}\left(\lambda_{0}\right)=b_{3}\left(\lambda_{0}\right) \neq 0
$$

The global relations (4.40) yield

$$
a_{2}\left(-\lambda_{0}\right)-1=b_{2}\left(-\lambda_{0}\right), \quad \mathrm{e}^{-\omega\left(\lambda_{0}\right) L}+a_{2}\left(\lambda_{0}\right)=b_{2}\left(\lambda_{0}\right) .
$$

Using these equations to eliminate $b_{2}(\lambda)$ and $b_{2}(-\lambda)$ from the determinant condition

$$
a_{2}\left(\lambda_{0}\right) a_{2}\left(-\lambda_{0}\right)-b_{2}\left(\lambda_{0}\right) b_{2}\left(-\lambda_{0}\right)=1,
$$

we find

$$
a_{2}\left(\lambda_{0}\right)-e^{-\omega\left(\lambda_{0}\right) L} a_{2}\left(-\lambda_{0}\right)=1-e^{-\omega\left(\lambda_{0}\right) L} .
$$

If $\lambda_{0}$ is a zero of $a_{3}(\lambda)-b_{3}(\lambda)$, then, by (4.17), so is $-1 / \lambda_{0}$, and hence we also have

$$
a_{2}\left(-\frac{1}{\lambda_{0}}\right)-e^{\omega\left(\lambda_{0}\right) L} a_{2}\left(\frac{1}{\lambda_{0}}\right)=1-e^{\omega\left(\lambda_{0}\right) L} .
$$

On the other hand, the symmetry condition (4.26) for $a_{2}(\lambda)$ implies that

$$
a_{2}\left(\frac{1}{\lambda}\right)+e^{-\omega(\lambda) L} a_{2}\left(-\frac{1}{\lambda}\right)=a_{2}(\lambda)+e^{-\omega(\lambda) L} a_{2}(-\lambda), \quad \lambda \in \mathbb{C} .
$$

Indeed, if we use (4.26) to eliminate $a_{2}( \pm 1 / \lambda)$ from the left-hand side of (5.10) and then simplify, we find the right-hand side of (5.10). Evaluating (5.10) at $\lambda=\lambda_{0}$ and using (5.8) and (5.9) to eliminate $a_{2}\left(-1 / \lambda_{0}\right)$ and $a_{2}\left(-\lambda_{0}\right)$ from the resulting equation, we find

$$
a_{2}\left(\lambda_{0}\right)=a_{2}\left(\frac{1}{\lambda_{0}}\right)
$$

In view of (5.7), (5.8), and (5.11), the symmetry equation (4.26) for $a_{2}(\lambda)$ evaluated at $\lambda=\lambda_{0}$ reduces to

$$
0=-1-e^{-\omega\left(\lambda_{0}\right) L}+F\left(\lambda_{0}\right)\left(1-e^{-\omega\left(\lambda_{0}\right) L}\right), \quad \text { i.e. } \quad G\left(\lambda_{0}\right)=1 .
$$

This shows that $1-G(\lambda)=0$ whenever $a_{3}(\lambda)-b_{3}(\lambda)=0$. A similar argument shows that $1-G(\lambda)=0$ also whenever $a_{3}(-\lambda)+b_{3}(-\lambda)=0$. This proves $(d)$. The proof of $(e)$ is similar. The statement $(f)$ follows from $(d)$ and $(e)$ since $h=\left(a_{3}-b_{3}\right)\left(a_{3}+b_{3}\right)$.

Since $h(\lambda)$ is analytic in $\mathbb{C}^{+}$and $h(\lambda)$ does not vanish at any point $\lambda \in P_{G}$ by $(f)$, equation (5.6) implies that $h(-\lambda)$ has a double pole at each point in the set $P_{G} \cap \mathbb{C}^{+}$. This proves $(g)$. QED 


\subsection{An equivalent Riemann-Hilbert problem}

Using the relations (4.16) and (4.32a), the jump matrices (2.26) become

$$
\begin{gathered}
J^{0}(x, y, \lambda)=\left(\begin{array}{cc}
1+\frac{b_{3}(\lambda) b_{3}(-\lambda)}{a_{3}(\lambda) a_{3}(-\lambda)} \mathrm{e}^{-\omega(\lambda) L} & \frac{b_{3}(-\lambda)}{a_{3}(\lambda)} \mathrm{e}^{-\theta(x, y, \lambda)} \\
\frac{b_{3}(\lambda)}{a_{3}(-\lambda)} \mathrm{e}^{-\omega(\lambda) L} \mathrm{e}^{\theta(x, y, \lambda)} & 1
\end{array}\right), \\
J^{\pi / 2}(x, y, \lambda)=\left(\begin{array}{cc}
1 & \frac{b_{2}(-\lambda)}{a_{3}(\lambda)^{2}} \mathrm{e}^{-\theta(x, y, \lambda)} \\
0 & 1
\end{array}\right), \quad J^{3 \pi / 2}(x, y, \lambda)=\left(\begin{array}{cc}
1 & 0 \\
\frac{b_{2}(\lambda)}{a_{3}(-\lambda)^{2}} \mathrm{e}^{\theta(x, y, \lambda)} & 1
\end{array}\right),
\end{gathered}
$$

and

$$
J^{\pi}=J^{3 \pi / 2}\left(J^{0}\right)^{-1} J^{\pi / 2}, \quad \mathrm{e}^{\theta(x, y, \lambda)}=\mathrm{e}^{\Omega(\lambda) x+\omega(\lambda) y} .
$$

Let $M(x, y, \lambda)$ be defined by $(2.24)$ with $\Psi_{j}$ replaced with $\Phi_{j}, j=1,2,3$. Let $D_{j}$ denote the $j$ th quadrant of the complex plane,

$$
D_{j}=\left\{\lambda \in \mathbb{C} \mid(j-1) \frac{\pi}{2}<\arg (\lambda)<j \frac{\pi}{2}\right\}, \quad j=1, \ldots, 4,
$$

and let $M_{j}$ denote the restriction of $M$ to $D_{j}$.

The jump matrices (5.12) involve the unknown spectral functions $b_{2}(\lambda), a_{3}(\lambda)$, and $b_{3}(\lambda)$. We therefore seek matrices $A_{j}(x, y, \lambda), j=1, \ldots, 4$, defined for $\lambda \in D_{j}$, such that the functions $\left\{\tilde{M}_{j}(x, y, \lambda)\right\}_{1}^{4}$ by

$$
\tilde{M}_{j}(x, y, \lambda)=M_{j}(x, y, \lambda) A_{j}(x, y, \lambda), \quad \lambda \in D_{j}, \quad j=1, \ldots, 4,
$$

satisfy a modified Riemann-Hilbert problem whose jump matrices involve only known functions. We would like $A_{j}$ to be bounded and analytic (or at least meromorphic) for $\lambda \in D_{j}$.

The requirement that $A_{j}$ is bounded in the $j$-th quadrant implies that $A_{1}$ and $A_{2}$ are upper triangular, while $A_{3}$ and $A_{4}$ are lower triangular. The requirement that $A_{j}$ has unit determinant implies that the diagonal elements of $A_{j}$ are $d_{j}$ and $\frac{1}{d_{j}}$. The $(2,2)$ components of equations $(5.18)$ imply that

$$
d_{1}=d_{2}=d_{3}=d_{4}
$$

On the other hand, the four exponential factors

$$
\mathrm{e}^{-\theta(x, y, \lambda)}, \quad \mathrm{e}^{-\theta(x, y, \lambda)} \mathrm{e}^{\omega(\lambda) L}, \quad \mathrm{e}^{\theta(x, y, \lambda)}, \quad \mathrm{e}^{\theta(x, y, \lambda)} \mathrm{e}^{-\omega(\lambda) L}
$$

are bounded in the first, second, third and fourth quadrant of the complex $\lambda$-plane, respectively. This suggests choosing the matrices $A_{j}$ in the following form:

$$
\begin{aligned}
& A_{1}=\left(\begin{array}{cc}
1 & \alpha_{1}(\lambda) \mathrm{e}^{-\theta(x, y, \lambda)} \\
0 & 1
\end{array}\right), \quad \lambda \in D_{1}, \\
& A_{2}=\left(\begin{array}{cc}
1 & \alpha_{2}(\lambda) \mathrm{e}^{\omega(\lambda) L} \mathrm{e}^{-\theta(x, y, \lambda)} \\
0 & 1
\end{array}\right), \quad \lambda \in D_{2},
\end{aligned}
$$




$$
\begin{aligned}
& A_{3}=\left(\begin{array}{cc}
1 & 0 \\
\alpha_{3}(\lambda) \mathrm{e}^{\theta(x, y, \lambda)} & 1
\end{array}\right), \quad \lambda \in D_{3}, \\
& A_{4}=\left(\begin{array}{cc}
1 & 0 \\
\alpha_{4}(\lambda) \mathrm{e}^{-\omega(\lambda) L} \mathrm{e}^{\theta(x, y, \lambda)} & 1
\end{array}\right), \quad \lambda \in D_{4} .
\end{aligned}
$$

where $\alpha_{j}(\lambda), j=1, \ldots, 4$, is a scalar valued function of $\lambda \in D_{j}$.

Substituting (5.13) into the jump relations

$$
M_{4}=M_{1} J^{0}, \quad M_{2}=M_{1} J^{\pi / 2}, \quad M_{4}=M_{3} J^{3 \pi / 2},
$$

we find the equations

$$
\tilde{M}_{4}=\tilde{M}_{1} \tilde{J}^{0}, \quad \tilde{M}_{2}=\tilde{M}_{1} \tilde{J}^{\pi / 2}, \quad \tilde{M}_{4}=\tilde{M}_{3} \tilde{J}^{3 \pi / 2},
$$

provided that the matrices $\tilde{J}^{0}, \tilde{J}^{\pi / 2}, \tilde{J}^{3 \pi / 2}$ satisfy the following equations:

$$
J^{0} A_{4}=A_{1} \tilde{J}^{0}, \quad J^{\pi / 2} A_{2}=A_{1} \tilde{J}^{\pi / 2}, \quad J^{3 \pi / 2} A_{4}=A_{3} \tilde{J}^{3 \pi / 2} .
$$

We analyse the first of equations (5.18). The $(2,2)$ element of this equation is satisfied identically and the $(1,1)$ element is a consequence of the $(1,2)$ and $(2,1)$ elements, as well as of the requirement that all matrices in $(5.18)$ have unit determinant. Denoting the $(1,2)$ and $(2,1)$ components of $\tilde{J}^{0}$ by $\mathrm{e}^{-\theta(x, y, \lambda)} \tilde{U}^{0}(\lambda)$ and $\mathrm{e}^{\theta(x, y, \lambda)} \mathrm{e}^{-\omega(\lambda) L} \tilde{V}^{0}(\lambda)$ respectively, we find that the $(1,2)$ and $(2,1)$ elements of the first of equations (5.18) yield

$$
\frac{b_{3}(-\lambda)}{a_{3}(\lambda)}-\alpha_{1}(\lambda)=\tilde{U}^{0}(\lambda)
$$

and

$$
\frac{b_{3}(\lambda)}{a_{3}(-\lambda)}+\alpha_{4}(\lambda)=\tilde{V}^{0}(\lambda)
$$

Comparing equation (5.19) with the identity (5.1) we find that a simple choice for the function $\alpha_{1}$ (and hence for $\tilde{U}^{0}$ ) is $\alpha_{1}(\lambda)=\frac{b_{3}(\lambda)}{a_{3}(\lambda) h(\lambda)}$ and $\tilde{U}^{0}(\lambda)=-\frac{G(\lambda)}{h(\lambda)}$. Note that these functions are well defined on $\mathbb{R}$ since $h$ does not have any real zero. However, with these choices the functions $\alpha_{1}(\lambda)$ and $\tilde{U}^{0}(\lambda)$ have (i) poles at the (unknown) zeros of $h(\lambda)$ along the imaginary axis and (ii) poles at the (known) poles of $G(\lambda)$ along the imaginary axis. To ensure that the poles in (i) are removable singularities we define a function $\tilde{G}(\lambda)$ as follows:

$$
\tilde{G}(\lambda)=G(\lambda)(h(\lambda)+1) .
$$

This function takes value 1 and -1 exactly where $G(\lambda)$ does. Indeed, if $h(\lambda)=0$, then $a_{3}(\lambda)=$ $\pm b_{3}(\lambda)$ and correspondingly, in view of (5.5), $G(\lambda)=\tilde{G}(\lambda)= \pm 1$.

The above discussion suggests that a suitable choice for the functions $\alpha_{1}(\lambda)$ and $\tilde{U}^{0}(\lambda)$ is

$$
\alpha_{1}(\lambda)=\frac{b_{3}(\lambda)}{a_{3}(\lambda) h(\lambda)}-\frac{\tilde{G}(\lambda)}{h(\lambda)}, \quad \tilde{U}^{0}(\lambda)=-\frac{G(\lambda)-\tilde{G}(\lambda)}{h(\lambda)}=G(\lambda) .
$$


Similarly, equation (5.20) suggests

$$
\alpha_{4}(\lambda)=-\frac{b_{3}(-\lambda)}{a_{3}(-\lambda) h(-\lambda)}+\frac{\tilde{G}(-\lambda)}{h(-\lambda)}, \quad \tilde{V}^{0}(\lambda)=-\frac{G(-\lambda)-\tilde{G}(-\lambda)}{h(-\lambda)}=-G(\lambda) .
$$

We next analyse the second of equations $(5.18)$. The $(1,2)$ element of this equation yields

$$
\alpha_{2}(\lambda) \mathrm{e}^{\omega(\lambda) L}+\frac{b_{2}(-\lambda)}{a_{3}(\lambda)^{2}}=\frac{b_{3}(\lambda)}{a_{3}(\lambda) h(\lambda)}-\frac{\tilde{G}(\lambda)}{h(\lambda)}+\tilde{U}^{\pi / 2}(\lambda)
$$

where $\tilde{U}^{\pi / 2}(\lambda) \mathrm{e}^{-\theta(x, y, \lambda)}$ denotes the $(1,2)$ component of $\tilde{J}^{\pi / 2}$. Using the identity (5.2), we find

$$
\alpha_{2} \mathrm{e}^{\omega(\lambda) L}+\mathrm{e}^{\omega(\lambda) L} \frac{b_{3}(\lambda)}{a_{3}(\lambda) h(\lambda)}-\left(1+\mathrm{e}^{\omega(\lambda) L}\right) \frac{G(\lambda)}{h(\lambda)}+\frac{\tilde{G}(\lambda)}{h(\lambda)}=\tilde{U}^{\pi / 2}(\lambda) .
$$

This suggests that we define

$$
\alpha_{2}(\lambda)=-\frac{b_{3}(\lambda)}{a_{3}(\lambda) h(\lambda)}+\frac{\tilde{G}(\lambda)}{h(\lambda)}, \quad \tilde{U}^{\pi / 2}(\lambda)=\left(1+\mathrm{e}^{\omega(\lambda) L}\right) G(\lambda) .
$$

A similar analysis of the third of equations (5.18) yields

$$
\alpha_{3}(\lambda)=\frac{b_{3}(-\lambda)}{a_{3}(-\lambda) h(-\lambda)}-\frac{\tilde{G}(-\lambda)}{h(-\lambda)}, \quad \tilde{V}^{3 \pi / 2}(\lambda)=\left(1+\mathrm{e}^{-\omega(\lambda) L}\right) G(-\lambda),
$$

where $V^{3 \pi / 2}(\lambda) \mathrm{e}^{\theta(x, y, \lambda)}$ denotes the $(2,1)$ component of $\tilde{J}^{\frac{3 \pi}{2}}$. Note that the relations $\alpha_{2}(\lambda)=$ $\alpha_{4}(-\lambda)$ and $\alpha_{3}(\lambda)=\alpha_{1}(-\lambda)$ are consistent with the symmetry $(2.20)$.

We define the matrices $A_{j}, j=1, \ldots, 4$, by equations (5.15) and (5.22)-(5.25). Henceforth, we assume that $(x, y)$ lies in the interior of the semistrip (1.2) so that $x>0$ and $0<y<L$. Then the $j$ th exponential factor in (5.14) has exponential decay as $\lambda \rightarrow \infty$ and $\lambda \rightarrow 0$ for $\lambda \in \bar{I}_{j}$. Thus, although the analysis of the linear problem suggests that the spectral functions $a_{j}(\lambda), b_{j}(\lambda)$ could have some minor growth as $\lambda \rightarrow \infty$ and $\lambda \rightarrow 0$ caused by the jumps in the boundary data at the corners of the semistrip (in the linear case this growth is logarithmic, see Appendix A), this ensures that the $A_{j}$ 's are bounded as $\lambda \rightarrow \infty$ and as $\lambda \rightarrow 0$ in the corresponding domains $D_{j}$.

In fact, since the $A_{j}$ 's have removable singularities at the zeros of the function $h(\lambda)$ along the imaginary axis, the only remaining difficulty is that the $A_{j}$ 's have singularities at the known poles of $G(\lambda)$. To deal with these singularities, we add small indentations to the jump contour along the imaginary axis so that it passes to the right of the poles of $G$. Thus, instead of the four quadrants $D_{j}$ of the complex plane, we consider the deformed domains $\tilde{D}_{j}$ defined in such a way that all $\lambda \in P_{G} \cap \mathbb{C}^{+}$lie in $\tilde{D}_{1}$ and all $\lambda \in P_{G} \cap \mathbb{C}^{-}$lie in $\tilde{D}_{3}$, see Figure 5 .

We next determine the residue conditions at these poles. Let $\lambda^{*} \in P_{G} \cap \mathbb{C}^{+}$be a pole of $\alpha_{1}$ in $\tilde{D}_{1}$. In what follows, we use the notation $M(x, y, \lambda)=\left([M(x, y, \lambda)]_{1},[M(x, y, \lambda)]_{2}\right)$ to denote the first and second column vector of a given matrix $M(x, y, \lambda)$. Then the relation $\tilde{M}_{1}=M_{1} A_{1}$ implies that

$$
\left[\tilde{M}_{1}(x, y, \lambda)\right]_{1}=\left[M_{1}(x, y, \lambda)\right]_{1}, \quad\left[\tilde{M}_{1}(x, y, \lambda)\right]_{2}=\alpha_{1}(\lambda) e^{-\theta(x, y, \lambda)}\left[M_{1}(x, y, \lambda)\right]_{1}+\left[M_{1}(x, y, \lambda)\right]_{2} .
$$




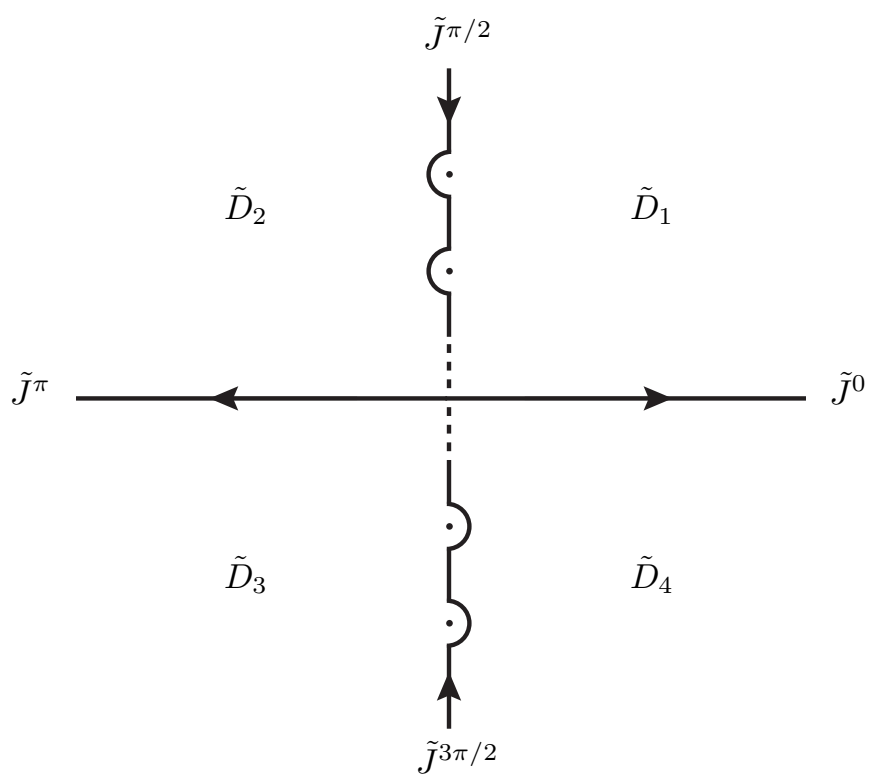

Figure 5: The contour for the modified Riemann-Hilbert problem satisfied by $\tilde{M}$. The contour has small indentations bypassing the poles of $G(\lambda)$.

Taking the residue of the second of these equations at $\lambda^{*}$, we find

$$
\begin{aligned}
\operatorname{Res}_{\lambda^{*}}\left[\tilde{M}_{1}(x, y, \lambda)\right]_{2} & =\left(\operatorname{Res}_{\lambda^{*}} \alpha_{1}\right) e^{-\theta\left(x, y, \lambda^{*}\right)}\left[M_{1}\left(x, y, \lambda^{*}\right)\right]_{1} \\
& =-\left(\operatorname{Res}_{\lambda^{*}} G\right)\left(1+\frac{1}{h\left(\lambda^{*}\right)}\right) e^{-\theta\left(x, y, \lambda^{*}\right)}\left[\tilde{M}_{1}\left(x, y, \lambda^{*}\right)\right]_{1},
\end{aligned}
$$

where the residue of $G(\lambda)$ at $\lambda^{*}$ is known from the definition (4.30) whereas the number $h\left(\lambda^{*}\right)$ remains unknown.

Similarly, the relation $\tilde{M}_{3}=M_{3} A_{3}$ implies that

$$
\left[\tilde{M}_{3}(x, y, \lambda)\right]_{1}=\left[M_{3}(x, y, \lambda)\right]_{1}+\alpha_{3}(\lambda) e^{\theta(x, y, \lambda)}\left[M_{3}(x, y, \lambda)\right]_{2}, \quad\left[\tilde{M}_{3}(x, y, \lambda)\right]_{2}=\left[M_{3}(x, y, \lambda)\right]_{2} .
$$

Taking the residue of the first of these equations at $\lambda^{*} \in P_{G} \cap \mathbb{C}^{-}$, we find

$$
\begin{aligned}
\operatorname{Res}_{\lambda^{*}}\left[\tilde{M}_{3}(x, y, \lambda)\right]_{1} & =\left(\operatorname{Res}_{\lambda^{*}} \alpha_{3}\right) e^{\theta\left(x, y, \lambda^{*}\right)}\left[M_{3}\left(x, y, \lambda^{*}\right)\right]_{2} \\
& =\left(\operatorname{Res}_{\lambda^{*}} G\right)\left(1+\frac{1}{h\left(-\lambda^{*}\right)}\right) e^{\theta\left(x, y, \lambda^{*}\right)}\left[\tilde{M}_{3}\left(x, y, \lambda^{*}\right)\right]_{2},
\end{aligned}
$$

In summary, we have derived the following result.

Theorem 5.1 The RH problem defined in theorem 3.1 and characterized by the jump matrices $\left\{J^{\pi / 2}, J^{3 \pi / 2}, J^{0}, J^{\pi}\right\}$ defined in equation (5.12), can be mapped to a new RH problem with the 
following jump matrices:

$$
\begin{aligned}
\tilde{J}^{\pi / 2}(x, y, \lambda) & =\left(\begin{array}{cc}
1 & \left(1+\mathrm{e}^{\omega(\lambda) L}\right) G(\lambda) \mathrm{e}^{-\theta(x, y, \lambda)} \\
0 & 1
\end{array}\right), \\
\tilde{J}^{3 \pi / 2}(x, y, \lambda) & =\left(\begin{array}{cc}
1 & 0 \\
-\left(1+\mathrm{e}^{-\omega(\lambda) L}\right) G(\lambda) \mathrm{e}^{\theta(x, y, \lambda)} & 1
\end{array}\right), \\
\tilde{J}^{0}(x, y, \lambda) & =\left(\begin{array}{cc}
1-G^{2}(\lambda) \mathrm{e}^{-\omega(\lambda) L} & G(\lambda) \mathrm{e}^{-\theta(x, y, \lambda)} \\
-G(\lambda) \mathrm{e}^{-\omega(\lambda) L} \mathrm{e}^{\theta(x, y, \lambda)} & 1
\end{array}\right),
\end{aligned}
$$

where the known function $G(\lambda)$ is defined in (4.30). This is achieved by using the matrices (5.15), with

$$
\begin{aligned}
\alpha_{1}(\lambda) & =\frac{b_{3}(\lambda)}{a_{3}(\lambda) h(\lambda)}-\frac{\tilde{G}(\lambda)}{h(\lambda)}, & \alpha_{2}(\lambda) & =-\frac{b_{3}(\lambda)}{a_{3}(\lambda) h(\lambda)}+\frac{\tilde{G}(\lambda)}{h(\lambda)}, \\
\alpha_{3}(\lambda) & =\alpha_{1}(-\lambda), & \alpha_{4}(\lambda) & =\alpha_{2}(-\lambda) .
\end{aligned}
$$

where the functions $h(\lambda)$ and $\tilde{G}(\lambda)$ are defined by (5.3) and (5.21) respectively. The solution $\tilde{M}$ of the new RH problem is a sectionally meromorphic function with simple poles at each point in the set $P_{G}$ given in (5.4). At these points the following residue conditions are valid:

$$
\begin{array}{cl}
\operatorname{Res}_{\lambda^{*}}[\tilde{M}(x, y, \lambda)]_{2}=-\left(\operatorname{Res}_{\lambda^{*}} G\right)\left(1+\frac{1}{h\left(\lambda^{*}\right)}\right) e^{-\theta\left(x, y, \lambda^{*}\right)}\left[\tilde{M}\left(x, y, \lambda^{*}\right)\right]_{1}, & \lambda^{*} \in P_{G} \cap \mathbb{C}^{+}, \\
\operatorname{Res}_{\lambda^{*}}[\tilde{M}(x, y, \lambda)]_{1}=\left(\operatorname{Res}_{\lambda^{*}} G\right)\left(1+\frac{1}{h\left(-\lambda^{*}\right)}\right) e^{\theta\left(x, y, \lambda^{*}\right)}\left[\tilde{M}\left(x, y, \lambda^{*}\right)\right]_{2}, & \lambda^{*} \in P_{G} \cap \mathbb{C}^{-} .
\end{array}
$$

The solution $q(x, y), x>0,0<y<L$, of the boundary value problem determined by the boundary conditions (1.3) is given by

$$
\begin{aligned}
\left(q_{x}-i q_{y}\right)(x, y) & =2 \lim _{\lambda \rightarrow \infty} \lambda(\tilde{M})_{12}, \\
\cos q(x, y) & =1+4 i\left(\lim _{\lambda \rightarrow \infty} \lambda\left(\tilde{M}_{11}\right)_{x}+2 \lim _{\lambda \rightarrow \infty} \lambda(\tilde{M})_{12}\right)^{2} .
\end{aligned}
$$

Proof: We only need to show how to represent the solution $q(x, y)$ of the boundary value problem in terms of the solution of the RH problem characterised by the jump matrices given by (5.26). Recall that if $M(x, y, \lambda)$ denotes the solution of the RH problem defined in theorem 3.1 then $q(x, y)$ is defined in terms of $M$ by equations (3.7)-(3.8). Using $\tilde{M}_{j}=A_{j} M_{j}$ in the $j$-th quadrant, $j=1, \ldots, 4$, by choosing $\lambda$ in the first quadrant we obtain

$$
\begin{aligned}
\left(q_{x}-i q_{y}\right)(x, y) & =2 \lim _{\lambda \rightarrow \infty} \lambda(\tilde{M})_{12} \\
\cos q(x, y) & =1+4 i\left(\lim _{\lambda \rightarrow \infty} \lambda\left[\tilde{M}_{11}-\alpha_{1}(\lambda) \mathrm{e}^{-\theta(x, y, \lambda)}(\tilde{M})_{21}\right]_{x}+2 \lim _{\lambda \rightarrow \infty} \lambda(\tilde{M})_{12}\right)^{2} .
\end{aligned}
$$

Since $\mathrm{e}^{-\theta(x, y, \lambda)}$ decays exponentially as $\lambda \rightarrow \infty$ in the first quadrant, the term involving the unknown coefficient $\alpha_{1}$ does not contribute to the limit and we find (5.30)-(5.31). 


\section{Conclusions and open problems}

We have analyzed the elliptic sine-Gordon equation in a semistrip for general boundary data (sections 2 and 3 ) and in the particular case of a linearisable boundary value problem (sections 4 and 5). The linearizable problem has the novelty that the function $q_{y}(x, 0)$ possesses a nonintegrable singularity as $x \rightarrow 0$ while the function $q_{x}(0, y)$ possesses a non-integrable singularity as $y \rightarrow 0$. Motivated by the recent solution of an analogous problem for the modified Helmholtz equation presented in [2], we have been able to bypass this problem by employing a suitable gauge transformation. Furthermore, we have shown that the RH problem characterizing the solution $q(x, y)$ can be mapped to a modified $\mathrm{RH}$ problem whose "jump matrix" is determined only by the width $L$ of the semistrip and the given constant value $d$ of the boundary condition prescribed at $x=0$ (see theorem 5.1). However, the modified $\mathrm{RH}$ problem also includes residue conditions at the points $\lambda \in P_{G}$, where the set $P_{G}$ consists of a countable number of points on the imaginary axis. The formulation of these residue conditions requires the knowledge of $h(\lambda)$ for $\lambda \in P_{G}$, where $h(\lambda)$ is an unknown meromorphic function defined in terms of the spectral functions. It remains an open problem to characterize the values of $h(\lambda)$ for $\lambda \in P_{G}$ in terms of $L$ and $d$ alone; progress in this direction is likely to rely on the analyticity properties of $h(\lambda)$ as well as on relations derived from the symmetry properties of the spectral functions, such as the relation (5.6), and the known structure of the poles of $h$.

\section{Acknowledgements}

This research was partially supported by EPSRC grant EP/E022960/1. ASF would like to express his gratitude to the Guggenheim Foundation, USA.

\section{A The modified Helmholtz equation}

The basic differential form associated with the modified Helmholtz equation

$$
\frac{\partial^{2} u(x, y)}{\partial x^{2}}+\frac{\partial^{2} u(x, y)}{\partial y^{2}}-u(x, y)=0
$$

is given by

$$
W_{B}(x, y, \lambda)=\frac{\mathrm{e}^{-\Omega(\lambda) x-\omega(\lambda) y}}{4}\left[i\left(u_{x}-i u_{y}-\frac{i u}{\lambda}\right) d x-\left(u_{x}-i u_{y}+\frac{i u}{\lambda}\right) d y\right],
$$

where $\Omega(\lambda)$ and $\omega(\lambda)$ are defined in (2.4). Indeed, it can be verified that

$$
d W_{B}=-\frac{\mathrm{e}^{-\Omega(\lambda) x-\omega(\lambda) y}}{4}\left[u_{x x}+u_{y y}-u\right] d x \wedge d y .
$$

Suppose that $u_{y}(x, y)$ has non-integrable singularities at $(0,0)$ and $(0, L)$. In order to eliminate these singularities we consider the differential form

$$
W(x, y, \lambda)=W_{B}(x, y, \lambda)-d\left(\mathrm{e}^{-\Omega(\lambda) x-\omega(\lambda) y} \kappa(x, y, \lambda)\right)
$$




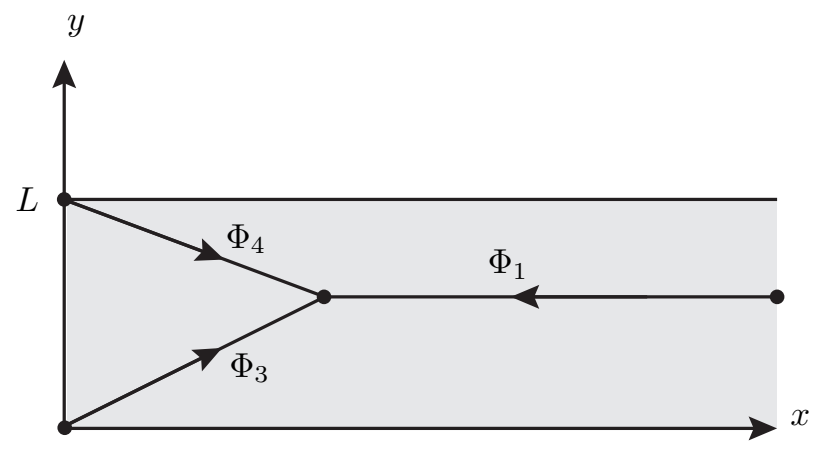

Figure 6: The contours of integration used to define $\Phi_{j}(x, y, \lambda), j=1,3,4$.

and choose $\kappa$ in such a way that the term $u_{y}$ cancels. Noting that

$$
W=\frac{\mathrm{e}^{-\Omega x-\omega y}}{4}\left\{\left[i u_{x}+u_{y}+\frac{u}{\lambda}-4 \kappa_{x}+4 \Omega \kappa\right] d x-\left[u_{x}-i u_{y}+i \frac{u}{\lambda}+4 \kappa_{y}-4 \omega \kappa\right] d y\right\}
$$

we choose $\kappa$ as in (4.2):

$$
\kappa(x, y, \lambda)=-\frac{1}{4} \int_{x}^{\infty} u_{y}(\xi, y) d \xi
$$

Then

$W=\frac{\mathrm{e}^{-\Omega x-\omega y}}{4}\left\{\left[i u_{x}+\frac{u}{\lambda}-\Omega \int_{x}^{\infty} u_{y}(\xi, y) d \xi\right] d x+\left[i u_{y}-i \frac{u}{\lambda}+\int_{x}^{\infty} u(\xi, y) d \xi-\omega \int_{x}^{\infty} u_{y}(\xi, y) d \xi\right] d y\right\}$

where we have used

$$
\kappa_{y}=-\frac{1}{4} \int_{x}^{\infty} u_{y y}(\xi, y) d \xi=-\frac{1}{4}\left(\int_{x}^{\infty} u(\xi, y) d \xi+u_{x}(x, y)\right)
$$

We define $\Phi_{j}(x, y, \lambda), j=1,3,4$, as the solutions of the equation

$$
d\left(\Phi_{j} \mathrm{e}^{-\Omega x-\omega y}\right)=W, \quad j=1,3,4,
$$

with

$$
\Phi_{1}(\infty, y, \lambda)=0, \quad \Phi_{3}(0,0, \lambda)=0, \quad \Phi_{4}(0, L, \lambda)=0,
$$

see Figure 6.

The difference of any two of the above functions equals $\mathrm{e}^{\Omega x+\omega y} \rho(\lambda)$, where $\rho(\lambda)$ can be computed by evaluating the difference at any convenient point $(x, y)$. Hence

$$
\begin{aligned}
& \Phi_{4}(x, y, \lambda)-\Phi_{1}(x, y, \lambda)=-\mathrm{e}^{\Omega x+\omega y} \mathrm{e}^{-\omega L} B_{1}(\lambda), \quad \lambda \in \mathbb{C}^{+}, \\
& \Phi_{4}(x, y, \lambda)-\Phi_{3}(x, y, \lambda)=\mathrm{e}^{\Omega x+\omega y} B_{2}(\lambda), \quad \lambda \in \mathbb{C}, \\
& \Phi_{1}(x, y, \lambda)-\Phi_{3}(x, y, \lambda)=\mathrm{e}^{\Omega x+\omega y} B_{3}(\lambda), \quad \lambda \in \mathbb{C}^{+},
\end{aligned}
$$

where

$$
B_{1}(\lambda)=\Phi_{1}(0, L, \lambda), \quad B_{2}(\lambda)=\Phi_{4}(0,0, \lambda), \quad B_{3}(\lambda)=\Phi_{1}(0,0, \lambda)
$$


and the above choice for the domains of validity with respect to $\lambda$ in equations (A.5) will be justified below.

Equations (A.3) and the first of equations (A.4) imply that

$$
\Phi_{1}(x, y, \lambda)=-\frac{1}{4} \int_{x}^{\infty} \mathrm{e}^{\Omega(x-\xi)}\left[i u_{\xi}+\frac{u}{\lambda}-\Omega \int_{\xi}^{\infty} u_{y}\left(\xi^{\prime}, y\right) d \xi^{\prime}\right] d \xi, \quad \lambda \in \mathbb{C}^{+} .
$$

Hence the first and the third of equations (A.6) imply that

$$
\begin{aligned}
& B_{3}(\lambda)=-\frac{1}{4} \int_{0}^{\infty} \mathrm{e}^{-\Omega \xi}\left[i u_{\xi}(\xi, 0)+\frac{u(\xi, 0)}{\lambda}-\Omega \int_{\xi}^{\infty} u_{y}\left(\xi^{\prime}, 0\right) d \xi^{\prime}\right] d \xi, \lambda \in \mathbb{C}^{+}, \\
& B_{1}(\lambda)=-\frac{1}{4} \int_{0}^{\infty} \mathrm{e}^{-\Omega \xi}\left[i u_{\xi}(\xi, L)+\frac{u(\xi, L)}{\lambda}-\Omega \int_{\xi}^{\infty} u_{y}\left(\xi^{\prime}, L\right) d \xi^{\prime}\right] d \xi, \lambda \in \mathbb{C}^{+} .
\end{aligned}
$$

In order to compute $\Phi_{4}(0,0, \lambda)$ we compute $\Phi_{4}$ along the $y$-axis from $(0, L)$ to $(0,0)$ :

$$
\Phi_{4}(0,0, \lambda)=\frac{1}{4} \int_{L}^{0} \mathrm{e}^{-\omega \eta}\left[i u_{\eta}(0, \eta)-i \frac{u(0, \eta)}{\lambda}+\int_{0}^{\infty} u(\xi, \eta) d \xi-\omega \int_{0}^{\infty} u_{\eta}(\xi, \eta) d \xi\right] d \eta
$$

The last integral of this equation is given by

$$
\begin{gathered}
\frac{\omega}{4} \int_{0}^{L} \mathrm{e}^{-\omega \eta}\left[\partial_{\eta} \int_{0}^{\infty} u(\xi, \eta) d \xi\right] d \eta=\frac{\omega}{4}\left[\mathrm{e}^{-\omega L} \int_{0}^{\infty} u(\xi, L) d \xi-\int_{0}^{\infty} u(\xi, 0) d \xi\right] \\
+\frac{\omega^{2}}{4} \int_{0}^{L} \mathrm{e}^{-\omega \eta}\left(\int_{0}^{\infty} u(\xi, \eta) d \xi\right) d \eta
\end{gathered}
$$

Hence, using this equation, as well as the identity $\Omega^{2}+\omega^{2}=1$, equation (A.10) together with the second of equations (A.6) yields

$$
\begin{aligned}
B_{2}(\lambda) & =\frac{1}{4} \int_{0}^{L} \mathrm{e}^{-\omega \eta}\left[-i u_{\eta}(0, \eta)+\frac{i u(0, \eta)}{\lambda}-\Omega^{2} \int_{0}^{\infty} u(\xi, \eta) d \xi\right] d \eta \\
& +\frac{\omega}{4}\left[\mathrm{e}^{-\omega L} \int_{0}^{\infty} u(\xi, L) d \xi-\int_{0}^{\infty} u(\xi, 0) d \xi\right], \quad \lambda \in \mathbb{C} .
\end{aligned}
$$

Subtracting the second of equations (A.5) from the sum of the other two equations in (A.5) we find the global relation

$$
\mathrm{e}^{-\omega(\lambda) L} B_{1}(\lambda)-B_{3}(\lambda)+B_{2}(\lambda)=0, \quad \lambda \in \mathbb{C}^{+} .
$$

\section{A.1 Example}

Let

$$
u(x, 0)=u(x, L)=0,0<x<\infty ; \quad u(0, y)=d, 0<y<L .
$$

The expressions in (A.8) and (A.11) simplify as follows:

$$
B_{3}(\lambda)=\Omega(\lambda) \int_{0}^{\infty} \mathrm{e}^{-\Omega(\lambda) \xi} f_{3}(\xi) d \xi, \quad f_{3}(\xi)=\frac{1}{4} \int_{\xi}^{\infty} u_{y}\left(\xi^{\prime}, 0\right) d \xi^{\prime}, \quad \lambda \in \mathbb{C}^{+},
$$




$$
B_{2}(\lambda)=\frac{i d\left(1-\mathrm{e}^{-\omega(\lambda) L}\right)}{2\left(1+\lambda^{2}\right)}-\Omega^{2} \int_{0}^{L} \mathrm{e}^{-\omega(\lambda) \eta} f_{2}(\eta) d \eta, \quad f_{2}(\eta)=\frac{1}{4} \int_{0}^{\infty} u(\xi, \eta) d \xi, \quad \lambda \in \mathbb{C} .
$$

Note that $\omega( \pm i)=0$, thus the first term of the right hand side of equation (A.14) has removable singularities at $\pm i$.

The overall symmetry $u(x, y)=u(x, L-y)$ implies

$$
B_{1}(\lambda)=-B_{3}(\lambda), \quad \lambda \in \mathbb{C}^{+} .
$$

Thus the global relation (A.12) becomes

$$
B_{2}(\lambda)=\left(1+\mathrm{e}^{-\omega(\lambda) L}\right) B_{3}(\lambda), \quad \lambda \in \mathbb{C}^{+} .
$$

The only dependence of $B_{3}$ on $\lambda$ is through $\Omega(\lambda)$, which remains invariant under the transformation $\lambda \rightarrow-\frac{1}{\lambda}$, thus

$$
B_{3}(\lambda)=B_{3}\left(-\frac{1}{\lambda}\right), \quad \lambda \in \mathbb{C}^{+} .
$$

The second term of the right hand side of the first of equations (A.14) involves $\Omega^{2}(\lambda)$ and $\omega(\lambda)$, which are invariant under the transformation $\lambda \rightarrow \frac{1}{\lambda}$. Thus we find

$$
B_{2}(\lambda)-\frac{i d\left(1-\mathrm{e}^{-\omega(\lambda) L}\right)}{2\left(1+\lambda^{2}\right)}=B_{2}\left(\frac{1}{\lambda}\right)-\frac{i d \lambda^{2}\left(1-\mathrm{e}^{-\omega(\lambda) L}\right)}{2\left(1+\lambda^{2}\right)},
$$

therefore

$$
B_{2}\left(\frac{1}{\lambda}\right)=B_{2}(\lambda)-\frac{i d}{2} \frac{1-\lambda^{2}}{1+\lambda^{2}}\left(1-\mathrm{e}^{-\omega(\lambda) L}\right), \quad \lambda \in \mathbb{C} .
$$

In summary, taking into account that $B_{1}=-B_{3}$, it follows that the modified Helmholtz equation in the semistrip, with the boundary conditions (1.3), involves the two unknown spectral functions $B_{3}(\lambda)$ and $B_{2}(\lambda)$, defined in terms of the two unknown functions $f_{2}(\lambda)$ and $f_{3}(\lambda)$ by equations (A.13) and (A.14). These two spectral functions satisfy the global relation (A.16) as well as the symmetry relations (A.17) and (A.18).

In what follows, we will show that the unknown functions $B_{2}$ and $B_{3}$ yields a zero contribution to the representation of the solution $u(x, y)$. In order to prove this fact, we need the following identities, which are a consequence of equations (A.16)-(A.18):

$$
\begin{gathered}
B_{3}(\lambda)-B_{3}(-\lambda)=G_{1}(\lambda), \quad G_{1}(\lambda)=\frac{i d}{2} \frac{1-\lambda^{2}}{1+\lambda^{2}} \frac{\mathrm{e}^{\omega(\lambda) L}-1}{\mathrm{e}^{\omega(\lambda) L}+1}, \quad \lambda \in \mathbb{R}, \\
B_{2}(\lambda)-\left(1+\mathrm{e}^{-\omega(\lambda) L}\right) B_{3}(-\lambda)=G_{2}(\lambda), \quad G_{2}(\lambda)=\frac{i d}{2} \frac{1-\lambda^{2}}{1+\lambda^{2}}\left(1-\mathrm{e}^{-\omega(\lambda) L}\right), \quad \lambda \in \mathbb{C}^{-} .
\end{gathered}
$$

Indeed, letting $\lambda \rightarrow \frac{1}{\lambda}$ in the global relation (A.16) we find

$$
B_{2}\left(\frac{1}{\lambda}\right)=\left(1+\mathrm{e}^{-\omega(\lambda) L}\right) B_{3}\left(\frac{1}{\lambda}\right), \quad \lambda \in \mathbb{C}^{-} .
$$

Using in the above equation the symmetry relation (A.17) with $\lambda \rightarrow-\lambda$, as well as the symmetry relation (A.18), we find equation (A.20). Subtracting equation (A.20) from the global relation (A.16) we find equation (A.19). The functions $G_{1}(\lambda), G_{2}(\lambda)$ have removable singularities at $\lambda= \pm i$. 


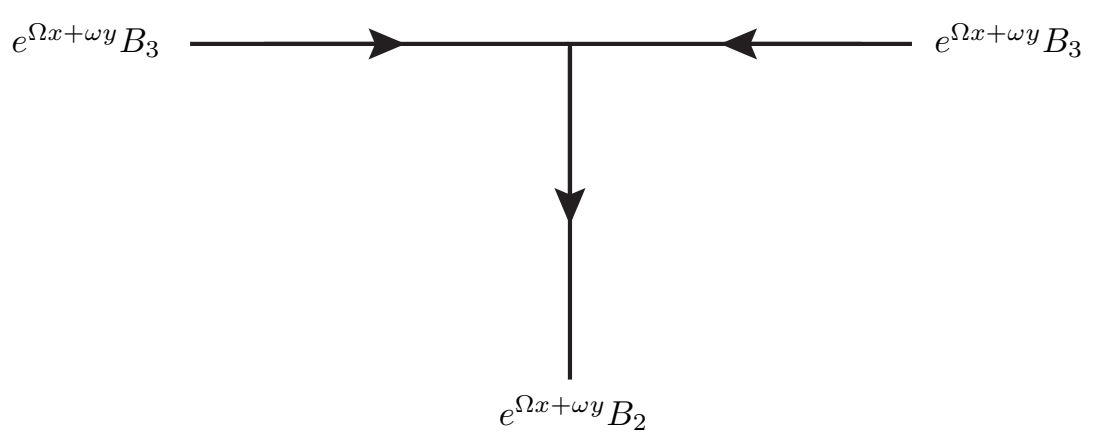

Figure 7: The contour for the Riemann-Hilbert problem.

The functions $\Phi_{j}(x, y, \lambda), j=1,3,4$, define a Riemann-Hilbert problem with jumps on the real and negative imaginary axis, see figure 7 .

In order to map this Riemann-Hilbert problem to a problem with known jump conditions, we introduce the functions $\tilde{\Phi}_{j}(x, y, \lambda), j=1,3,4$, through the following equations:

$$
\begin{array}{llrl}
\Phi_{1}=\tilde{\Phi}_{1}, & \lambda \in \mathbb{C}^{+}, \\
\Phi_{3}=\tilde{\Phi}_{3}-\mathrm{e}^{\theta(x, y, \lambda)} B_{3}(-\lambda), & \pi \leq \arg (\lambda) \leq \frac{3 \pi}{2}, \\
\Phi_{4}=\tilde{\Phi}_{4}+\mathrm{e}^{\theta(x, y, \lambda)} \mathrm{e}^{-\omega(\lambda) L} B_{3}(-\lambda), & \frac{3 \pi}{2} \leq \arg (\lambda) \leq 2 \pi .
\end{array}
$$

It is shown in remark A.1 at the end of this appendix that the function $B_{3}(\lambda)$ has a logarithmic singularity as $\lambda \rightarrow 0$ and $\lambda \rightarrow \infty$. In particular, assuming that $(x, y)$ lies in the interior of the semistrip (1.2) so that $x>0$ and $0<y<L$, it follows that $\mathrm{e}^{\theta(x, y, \lambda)} B_{3}(-\lambda)$ and $\mathrm{e}^{\theta(x, y, \lambda)} \mathrm{e}^{-\omega(\lambda) L} B_{3}(-\lambda)$ are bounded and analytic for $\lambda$ in the third and fourth quadrant of the $\lambda$ plane, respectively.

Using the definitions (A.21) in equation (A.5), we find

$$
\begin{aligned}
& \tilde{\Phi}_{4}(x, y, \lambda)-\tilde{\Phi}_{1}(x, y, \lambda)=\mathrm{e}^{\theta(x, y, \lambda)} \mathrm{e}^{-\omega(\lambda) L}\left(B_{3}(\lambda)-B_{3}(-\lambda)\right), \quad \lambda \in \mathbb{R}^{+}, \\
& \tilde{\Phi}_{4}(x, y, \lambda)-\tilde{\Phi}_{3}(x, y, \lambda)=\mathrm{e}^{\theta(x, y, \lambda)}\left(B_{2}(\lambda)-\left(1+\mathrm{e}^{-\omega(\lambda) L}\right) B_{3}(-\lambda)\right), \quad \lambda \in-i \mathbb{R}^{+}, \\
& \tilde{\Phi}_{1}(x, y, \lambda)-\tilde{\Phi}_{3}(x, y, \lambda)=\mathrm{e}^{\theta(x, y, \lambda)}\left(B_{3}(\lambda)-B_{3}(-\lambda)\right), \quad \lambda \in \mathbb{R}^{-} .
\end{aligned}
$$

Equations (A.19) and (A.20) imply that the jump conditions appearing in (A.22) can be expressed in terms of the known functions $G_{1}$ and $G_{2}$.

Equation (A.3) implies that the function $\Phi$ satisfies the equation

$$
\Phi_{x}-\Omega \Phi=\frac{1}{4}\left[i u_{x}+\frac{u}{\lambda}-\Omega \int_{x}^{\infty} u_{y}(\xi, y) d \xi\right]
$$

This equation suggests that

$$
\Phi_{1}(x, y, \lambda)=\frac{1}{4} \int_{x}^{\infty} u_{y}(\xi, y) d \xi+O\left(\frac{1}{\lambda}\right), \quad \lambda \rightarrow \infty .
$$


This estimate can be verified using equation (A.7) and integration by parts. The first of equations (A.21) shows that $\tilde{\Phi}_{1}$ satisfies the same estimate (A.24). Solving the Riemann-Hilbert problem with the jump conditions (A.22) and the estimate (A.24) (for $\tilde{\Phi}_{1}$ ) we find

$$
\begin{gathered}
\tilde{\Phi}(x, y, \lambda)=\frac{1}{4} \int_{x}^{\infty} u_{y}(\xi, y) d \xi+\frac{1}{2 \pi i}\left[\int_{-\infty}^{0} \mathrm{e}^{\Omega\left(\lambda^{\prime}\right) x+\omega\left(\lambda^{\prime}\right) y} G_{1}\left(\lambda^{\prime}\right) \frac{d \lambda^{\prime}}{\lambda^{\prime}-\lambda}\right. \\
\left.+\int_{\infty}^{0} \mathrm{e}^{\Omega\left(\lambda^{\prime}\right) x+\omega\left(\lambda^{\prime}\right)(y-L)} G_{1}\left(\lambda^{\prime}\right) \frac{d \lambda^{\prime}}{\lambda^{\prime}-\lambda}+\int_{0}^{-i \infty} \mathrm{e}^{\Omega\left(\lambda^{\prime}\right) x+\omega\left(\lambda^{\prime}\right) y} G_{2}\left(\lambda^{\prime}\right) \frac{d \lambda^{\prime}}{\lambda^{\prime}-\lambda}\right], \quad \lambda \in \mathbb{C}, \lambda \notin \mathbb{R} \cup i \mathbb{R} .
\end{gathered}
$$

Hence taking the limit of this equation as $\lambda \rightarrow 0$ we find

$$
\begin{gathered}
\lim _{\lambda \rightarrow 0}\left[\tilde{\Phi}(x, y, \lambda)-\frac{1}{4} \int_{x}^{\infty} u_{y}(\xi, y) d \xi\right]=\frac{1}{2 \pi i}\left[\int_{-\infty}^{0} \mathrm{e}^{\Omega(\lambda) x+\omega(\lambda) y} G_{1}(\lambda) \frac{d \lambda}{\lambda}\right. \\
\left.+\int_{\infty}^{0} \mathrm{e}^{\Omega(\lambda) x+\omega(\lambda)(y-L)} G_{1}(\lambda) \frac{d \lambda}{\lambda}+\int_{0}^{-i \infty} \mathrm{e}^{\Omega(\lambda) x+\omega(\lambda) y} G_{2}(\lambda) \frac{d \lambda}{\lambda}\right] .
\end{gathered}
$$

On the other hand, equation (A.23) implies that

$$
u(x, y)=-2 i \lim _{\lambda \rightarrow 0}\left[\Phi(x, y, \lambda)-\frac{1}{4} \int_{x}^{\infty} u_{y}(\xi, y) d \xi\right] .
$$

Noting that $\Phi=\tilde{\Phi}$ for $\lambda \in \mathbb{C}^{+}$, we find

$$
\begin{gathered}
u(x, y)=-\frac{1}{\pi}\left[\int_{-\infty}^{0} \mathrm{e}^{\Omega(\lambda) x+\omega(\lambda) y} G_{1}(\lambda) \frac{d \lambda}{\lambda}+\int_{\infty}^{0} \mathrm{e}^{\Omega(\lambda) x+\omega(\lambda)(y-L)} G_{1}(\lambda) \frac{d \lambda}{\lambda}+\right. \\
\left.+\int_{0}^{-i \infty} \mathrm{e}^{\Omega(\lambda) x+\omega(\lambda) y} G_{2}(\lambda) \frac{d \lambda}{\lambda}\right] .
\end{gathered}
$$

In summary, the solution of the BVP obtained by taking the linear limit of (1.1) and (1.3) is given by equation (A.27) where $G_{1}$ and $G_{2}$ are defined by (A.19) and (A.20).

In what follows we verify that the function $u(x, y)$ defined by (A.27) satisfies the given boundary conditions.

$\mathbf{u}(\mathbf{x}, \mathbf{0})=\mathbf{0}$

Evaluating equation (A.27) at $y=0$ we find

$$
u(x, 0)=-\frac{1}{\pi}\left[\int_{-\infty}^{0} \mathrm{e}^{\Omega(\lambda) x} G_{1}(\lambda) \frac{d \lambda}{\lambda}+\int_{\infty}^{0} \mathrm{e}^{\Omega(\lambda) x} \mathrm{e}^{-\omega(\lambda) L} G_{1}(\lambda) \frac{d \lambda}{\lambda}+\int_{0}^{-i \infty} \mathrm{e}^{\Omega(\lambda) x} G_{2}(\lambda) \frac{d \lambda}{\lambda}\right] .
$$

The integrands of the second and third integrals of the right hand side of (A.28) are bounded and analytic in the fourth quadrant of the complex $\lambda$ plane. In order to map the first integral to an integral whose integrand is also bounded and analytic $\mathrm{n}$ the fourth quadrant, we replace in the first integral $\lambda$ by $-\frac{1}{\lambda}$ :

$$
\int_{-\infty}^{0} \mathrm{e}^{\Omega(\lambda) x} G_{1}(\lambda) \frac{d \lambda}{\lambda}=-\int_{0}^{\infty} \mathrm{e}^{\Omega(\lambda) x} G_{1}(\lambda) \frac{d \lambda}{\lambda} .
$$


Then combining this term with the second integral we find an integral involving

$$
\left(1+\mathrm{e}^{-\omega(\lambda) L}\right) G_{1}=\mathrm{e}^{-\omega(\lambda) L}\left(1+\mathrm{e}^{\omega(\lambda) L}\right) G_{1} .
$$

Hence (A.28) becomes

$$
u(x, 0)=-\frac{i d}{2 \pi}\left[\int_{\infty}^{0}+\int_{0}^{-i \infty}\right] \mathrm{e}^{\Omega(\lambda) x} \frac{1-\lambda^{2}}{1+\lambda^{2}}\left(1-\mathrm{e}^{-\omega(\lambda) L}\right) \frac{d \lambda}{\lambda} .
$$

By Jordan's lemma, the right hand side of this equation vanishes $(\lambda=-i$ is a removable singularity) and hence $u(x, 0)=0$.

$\mathbf{u}(\mathbf{x}, \mathbf{L})=\mathbf{0}$

Evaluating equation (A.27) at $y=L$, we find

$$
u(x, L)=-\frac{1}{\pi}\left[\int_{-\infty}^{0} \mathrm{e}^{\Omega(\lambda) x} \mathrm{e}^{\omega(\lambda) L} G_{1}(\lambda) \frac{d \lambda}{\lambda}+\int_{\infty}^{0} \mathrm{e}^{\Omega(\lambda) x} G_{1}(\lambda) \frac{d \lambda}{\lambda}+\int_{0}^{-i \infty} \mathrm{e}^{\Omega(\lambda) x} \mathrm{e}^{\omega(\lambda) L} G_{2}(\lambda) \frac{d \lambda}{\lambda}\right] .
$$

The integrands of the first and third integrals of the right hand side of (A.29) are bounded and analytic in the third quadrant of the complex $\lambda$ plane. In order to map the second integral to an integral whose integrand is also bounded and analytic in the third quadrant, we replace in the second integral $\lambda$ by $-\frac{1}{\lambda}$ :

$$
\int_{\infty}^{0} \mathrm{e}^{\Omega(\lambda) x} G_{1}(\lambda) \frac{d \lambda}{\lambda}=\int_{-\infty}^{0} \mathrm{e}^{\Omega(\lambda) x} G_{1}(\lambda) \frac{d \lambda}{\lambda} .
$$

Then combining this term with the first integral we find an integral involving $\left(1+\mathrm{e}^{\omega(\lambda) L}\right) G_{1}$. Hence (A.29) becomes

$$
u(x, L)=-\frac{i d}{2 \pi}\left[\int_{-\infty}^{0}+\int_{0}^{-i \infty}\right] \mathrm{e}^{\Omega(\lambda) x} \frac{1-\lambda^{2}}{1+\lambda^{2}}\left(\mathrm{e}^{\omega(\lambda) L}-1\right) \frac{d \lambda}{\lambda} .
$$

By Jordan's lemma, the right hand side of this equation vanishes, hence $u(x, L)=0$.

$\mathbf{u}(\mathbf{0}, \mathbf{y})=\mathbf{d}$

Evaluating equation (A.27) at $x=0$, we find

$$
\begin{gathered}
u(0, y)=-\frac{i d}{2 \pi}\left[\int_{-\infty}^{0} \mathrm{e}^{\omega(\lambda) y} \frac{1-\lambda^{2}}{1+\lambda^{2}} \frac{\mathrm{e}^{\omega(\lambda) L}-1}{\mathrm{e}^{\omega(\lambda) L}+1} \frac{d \lambda}{\lambda}+\int_{\infty}^{0} \mathrm{e}^{\omega(\lambda)(y-L)} \frac{1-\lambda^{2}}{1+\lambda^{2}} \frac{\mathrm{e}^{\omega(\lambda) L}-1}{\mathrm{e}^{\omega(\lambda) L}+1} \frac{d \lambda}{\lambda}+\right. \\
\left.+\int_{0}^{-i \infty} \mathrm{e}^{\omega(\lambda) y} \frac{1-\lambda^{2}}{1+\lambda^{2}}\left(1-\mathrm{e}^{-\omega(\lambda) L}\right) \frac{d \lambda}{\lambda}\right] .
\end{gathered}
$$

The first and second terms of the integrand of the the third integral on the right hand side are analytic in the third and fourth quadrant of the $\lambda$ complex plane, respectively. Before considering these terms separately, in order to take care of the singularity at $\lambda=-i$ in the denominator, we deform the contour of integration of the third integral to the curve $L$ depicted in figure 8 . 


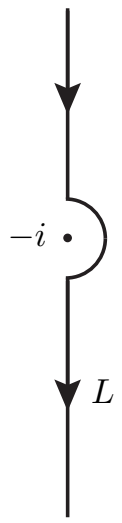

Figure 8: The contour for the Riemann-Hilbert problem.

Rewriting the term $\frac{\mathrm{e}^{\omega(\lambda) L}-1}{\mathrm{e}^{\omega(\lambda) L}+1}$ in the first and second integrals on the right hand side of (A.30) in the form, respectively,

$$
\frac{\mathrm{e}^{\omega(\lambda) L}-1}{\mathrm{e}^{\omega(\lambda) L}+1}=1-\frac{2}{\mathrm{e}^{\omega(\lambda) L}+1}=-1+\frac{2 \mathrm{e}^{\omega(\lambda) L}}{\mathrm{e}^{\omega(\lambda) L}+1}
$$

equation (A.30) becomes

$$
\begin{gathered}
u(0, y)=\frac{i d}{2 \pi}\left\{\left(\int_{\infty}^{0}+\int_{L}\right) \mathrm{e}^{\omega(\lambda)(y-L)} \frac{1-\lambda^{2}}{1+\lambda^{2}} \frac{d \lambda}{\lambda}+\right. \\
\left.-\left(\int_{-\infty}^{0}+\int_{L}\right) \mathrm{e}^{\omega(\lambda) y} \frac{1-\lambda^{2}}{1+\lambda^{2}} \frac{d \lambda}{\lambda}+2 \int_{-\infty}^{\infty} \frac{\mathrm{e}^{\omega(\lambda) y}}{1+\mathrm{e}^{\omega(\lambda) L}} \frac{1-\lambda^{2}}{1+\lambda^{2}} \frac{d \lambda}{\lambda}\right\} .
\end{gathered}
$$

Jordan's lemma implies that the first integral in the right hand side of (A.31) vanishes. Furthermore, the integrand of the third integral in the right hand side of (A.31) remains invariant under the transformation $\lambda \rightarrow \frac{1}{\lambda}$, thus this integral also vanishes. The second integral on the right hand side of (A.31) has a pole at $\lambda=-i$ with residue -1 . Hence, $u(0, y)=d$.

Remark A.1 - The asymptotics of $B_{3}(\lambda)$ as $\lambda \rightarrow 0$ and $\lambda \rightarrow \infty$

Using equation (A.27), it is possible to show (see [2]) that

$$
\frac{1}{4} \int_{x}^{\infty} u_{y}\left(x^{\prime}, 0\right) d x^{\prime}=-\frac{d}{2 \pi} \ln x+O(1), \quad x \rightarrow 0 .
$$

Hence, the definition (A.13) of $B_{3}(\lambda)$ implies that

$$
\begin{aligned}
B_{3}(\lambda)= & \Omega(\lambda) \int_{0}^{\infty} e^{-\Omega(\lambda) x}\left[\frac{1}{4} \int_{x}^{\infty} u_{y}\left(x^{\prime}, 0\right) d x^{\prime}+\frac{d}{2 \pi} \ln x\right] d x \\
& -\Omega(\lambda) \frac{d}{2 \pi} \int_{0}^{\infty} e^{-\Omega(\lambda) x} \ln x d x .
\end{aligned}
$$


Integration by parts implies that the first term of the rhs of (A.33) is of $O(1)$ as $\lambda \rightarrow \infty$ and $\lambda \rightarrow 0$. The second term in the rhs of equation (A.33) can be computed explicitly,

$$
\int_{0}^{\infty} e^{-\Omega(\lambda) x} \ln x d x=-\frac{\gamma+\ln \Omega}{\Omega}, \quad \operatorname{Re} \Omega>0 .
$$

Hence

$$
B_{3}(\lambda)=\frac{d}{2 \pi} \ln \Omega+O(1), \quad \lambda \rightarrow \infty \text { or } \lambda \rightarrow 0, \quad \lambda \in \mathbb{C}^{+} .
$$

In particular,

$$
B_{3}(\lambda)=\frac{d}{2 \pi} \ln \lambda+O(1), \quad \lambda \rightarrow \infty, \quad \lambda \in \mathbb{C}^{+},
$$

and

$$
B_{3}(\lambda)=-\frac{d}{2 \pi} \ln \lambda+O(1), \quad \lambda \rightarrow 0, \quad \lambda \in \mathbb{C}^{+}
$$

\section{References}

[1] Y.Antipov and A.S. Fokas, The modified Helmholtz equation in a semi-strip, Math. Proc. Camb. Phil Soc. 138, 339-365 (2005).

[2] A. Ashton and A.S. Fokas, The rigorous analysis of boundary value problems for linear elliptic PDEs, in preparation.

[3] D. ben-Avraham and A.S. Fokas, The Modified Helmholtz Equation in a Triangular Domain and an Application to Diffusion-limited Coalescence, Phys. Rev. E 64, 016114-6 (2001).

[4] J.L. Bona, and A.S. Fokas, Initial-Boundary-Value Problems for Linear and Integrable Nonlinear Dispersive Partial Differential Equations, Nonlinearity 21, 10, T195-T203 (2008).

[5] A. Boutet de Monvel, A.S. Fokas and D. Shepelsky, The Modified KdV Equation on the HalfLine, J. of the Inst. of Math. Jussieu 3, 139-164 (2004).

[6] A. Boutet de Monvel, A.S. Fokas and D. Shepelsky, The Analysis of the Global Relation for the Nonlinear Schrödinger Equation on the Half-Line, Lett. Math. Phys. 65, 199-212 (2003).

[7] A.B. Borisov and V.V. Kiseliev, Inverse problems for an elliptic sine-Gordon equation with an asymptotic behaviour of the cnoidal type, Inv. Prob. 5, 959-982 (1989).

[8] D. Crowdy and A.S. Fokas, Explicit Integral Solutions for the Plane Elastostatic Semi-Strip, Proc. R. Soc. London A 460, 1285-1309 (2004).

[9] G. Dassios and A.S. Fokas, The Basic Elliptic Equations in an Equilateral Triangle, Proc. R.Soc. Lond. A 461, 2721-2748 (2005).

[10] P. Deift, Orthogonal Polynomials and Random Matrices: A Riemann-Hilbert approach, American Mathematical Society (2000). 
[11] G.M. Dujardin, Asymptotics of Linear Initial Boundary Value Problems with Periodic Boundary Data on the Half-Line and Finite Intervals, Proc. R. Soc. Lond. A 465 3341-3360 (2009).

[12] N. Flyer and A.S. Fokas, A Hybrid Analytical Numerical Method for Solving Evolution Partial Differential Equations. I. The Half-Line, Proc. R. Soc. 464, 1823-1849 (2008).

[13] A.S. Fokas, A unified transform method for solving linear and certain nonlinear PDE's, Proc. Royal Soc. Series A 453, 1411-1443 (1997).

[14] A.S. Fokas, On the integrability of linear and nonlinear partial differential equations, J. Math. Phys, 41, 4188-4238 (2000).

[15] A.S. Fokas, A Unified Approach to Boundary Value Problems, CBMS-NSF Regional Conference Series in Applied Mathematics, SIAM (2008).

[16] A.S. Fokas, Two dimensional linear PDE's in a convex polygon, Proc. Royal Soc. Lond. A 457, 371-393 (2001).

[17] A.S. Fokas, Nonlinear evolution equations on the half line, Comm. Math. Phys. 230, 1-39 (2002).

[18] A.S. Fokas, The generalized Dirichlet-to-Neumann map for certain nonlinear evolution PDEs, Comm. Pure Appl. Math. LVIII, 639-670 (2005).

[19] A.S. Fokas, Linearizable initial boundary value problems for the sine-Gordon equation on the half line, Nonlin. 17, 1521-1534 (2004).

[20] A.S. Fokas, A New Transform Method for Evolution PDEs, IMA J. Appl. Math. 67, 1-32 (2002).

[21] A.S. Fokas and A.R. Its, The linearization of the initial-boundary value problem of the nonlinear Schrödinger equation, SIAM J. Math. Anal. 27 738-764 (1996).

[22] A.S. Fokas and A.R. Its, The nonlinear Schrödinger equation on the interval, J. Phys. A. 37, 6091-6114 (2004).

[23] A.S. Fokas, A.R. Its and L.Y. Sung, The Nonlinear Schrödinger Equation on the Half-Line, Nonlinearity 18, 1771-1822 (2005).

[24] A.S. Fokas and J. Lenells, Explicit Soliton Asymptotics for the Korteweg-de-Vries Equation on the Half-Line, Nonlin. 23, 937 (2010).

[25] A. S. Fokas and J. Lenells, The unified method: I Non-linearizable problems on the half-line, J. Phys. A: Math. Theor. 45, 195201.

[26] A.S. Fokas and C. Menyuk, Integrability and Similarity in Transient Stimulated Raman Scattering, J. Nonl. Sci. 9, 1-31 (1999).

[27] A.S. Fokas and B. Pelloni, A transform method for linear evolution PDEs on a finite interval, IMA J. of Appl. Math. 70, 1-24 (2005). 
[28] A.S. Fokas and E.A. Spence, Novel Analytical and Numerical Methods for Elliptic Boundary Value Problems, in "Highly Oscillatory Problems", Cambridge University Press (2009).

[29] A.S. Fokas and M. Zyskin, The Fundamental Differential Form and Boundary Value Problems, Quart. J. Mech. Appl. Math. 55, 457-479 (2002).

[30] E.S. Gutshabash and V.D.Lipovskii, Boundary value problem for the two-dimensional elliptic sine-Gordon equation and its applications to the theory of the stationary Josephson effect, $J$. Math. Sciences 68, 197-201 (1994).

[31] P. D. Lax, Integrals of nonlinear equations of evolution and solitary waves, Comm. Pure Appl. Math. 21, 467-490 (1968).

[32] J. Lenells, Boundary value problems for the stationary axisymmetric Einstein equations: a disk rotating around a black hole, Comm. Math. Phys. 304, 585635 (2011).

[33] J. Lenells and A.S. Fokas, An integrable generalisation of the nonlinear Schrödinger equation on the half-line and solitons, Inv. Prob. 25 (2009).

[34] J. Lenells and A.S. Fokas, Boundary Value Problems for the Stationary Axisymmetric Einstein Equations: A Rotating Disk, Nonlin 24, 177 (2011).

[35] J. Lenells and A. S. Fokas, The unified method: II NLS on the half-line with t-periodic boundary conditions, J. Phys. A: Math. Theor. 45, 195202.

[36] J. Lenells and A. S. Fokas, The unified method: III Non-linearizable problems on the interval, J. Phys. A: Math. Theor. 45, 195203.

[37] V.D. Lipovskii and S.S. Nikulichev, Vestn. LGU Ser. Fiz. Khim. 4, 61-34 (1988).

[38] B. Pelloni, The spectral representation of two-point boundary value problems for linear evolution equations, Proc. R. Soc. A (2005) 461, 2965-2984.

[39] B. Pelloni, Well posed boundary value problems for linear evolution equations in finite intervals, Math. Proc. Camb. Phil. Soc. (2004), 136, 361-382.

[40] B. Pelloni. The asymptotic behaviour of the solution of boundary value problems for the sine-Gordon equation on a finite interval, J. Nonlin. Math. Phys. 12(4), 518-529 (2005).

[41] B. Pelloni, Spectral analysis of the elliptic sine-Gordon equation in the quarter plane, Theor Math Phys. (2009) 160(1), 1031-1041.

[42] B. Pelloni and D.A. Pinotsis, The Klein-Gordon Equation on the Half Line: a Riemann-Hilbert Approach, J. Nonlinear Math. Phys. 15, 334 - 344 (2008).

[43] B. Pelloni and D.A. Pinotsis, The elliptic sine-Gordon equation in a half plane Nonlin. 23 $7788,(2010)$.

[44] S.A. Smitheman, E.A. Spence and A.S. Fokas, A Spectral Collocation Method for the Laplace and Modified Helmholtz Equations in a Convex Polygon, IMA J. Num. Anal. 30 1184-1205, (2010). 
[45] E.A. Spence, A.S. Fokas, A New Transform Method I: Domain Dependent Fundamental Solutions and Integral Representations, Proc. Roy. Soc. A 466 2259-2281 (2010).

[46] E.A. Spence, A.S. Fokas, A New Transform Method II: the Global Relation, and Boundary Value Problems in Polar Co-ordinates, Proc. Roy. Soc. A 466 2283-2307 (2010).

[47] P.A. Treharne and A.S. Fokas, The Generalized Dirichlet to Neumann map for the KdV Equation on the Half-Line, J. Nonlinear Science 18, 191-217 (2008).

[48] V.E. Zakharov and A.B.Shabat, A scheme for integrating the nonlinear equations of numerical physics by the method of the inverse scattering problem I, Funct. Anal. Appl. 8 226-235 (1974)

[49] V.E. Zakharov and A.B.Shabat, A scheme for integrating the nonlinear equations of numerical physics by the method of the inverse scattering problem II, Funct. Anal. Appl. 13 166-174 (1979)

[50] X. Zhou, The Riemann-Hilbert problem and inverse scattering, SIAM J. Math. Anal. 20 (4) 966-986 (1989). 